\title{
GEOGRAPHISCHER EXKURSIONSFÜHRER DER SCHWEIZ
}

\author{
Exkursionsraum Gempenplateau und benachbarte Talregionen
}

Hans Annaheim und Dietrich Barsch

\section{Vorbemerkung}

Nachdem G. Grosjean ein nach thematischen Kategorien gegliedertes Beispiel eines Exkursionsführers vorgelegt hatte (Exkursionsraum Bern-Längenberg-Schwarzenburg-Sensegraben, Geogr. Helv. 1961, 134-152), wurde von der Forschungskommission des Verbandes Schweiz. Geogr. Gesellschaften der Wunsch nach der Ausarbeitung eines Routenführers geäuBert, welcher die regionaltypischen Einblicke in den Exkursionsraum anhand einer «StandardExkursion» vermitteln sollte (vgl. dazu H. Annaheim, Geogr. Helv. 1961, 133 f.). Die vorliegende Bearbeitung kommt diesem Wunsche nach und soll den für das weitere Vorgehen grundsätzlich wichtigen Entscheid über die zu wählende Gestaltung des «Geographischen Exkursionsführers der Schweiz»erleichtern. Der Routenführer enthält wie das Beispiel Schwarzenburgerland zuerst Hinweise über das Ziel der Exkursion und einige technische Angaben und am Schluß eine knappe landschaftskundliche Synthese und den statistischen Anhang. Um jene Leser, welche dem Routenführer lediglich Informationen über ein bestimmtes Landschaftselement zu entnehmen wünschen, die Verwendung des Führers zu erleichtern, sind die betreffenden Stellen durch verschiedene Marginalien herausgehoben.

\section{ZIEL DER EXKURSION}

Einblick in die landschaftliche Struktur des unteren Birs- und Hochrheintales, des Gempenplateaus und seiner Abdachungen, in Landschaften also, die sich naturräumlich erheblich voneinander unterscheiden und deren kulturräumliche Differenzierung durch die neuere Entwicklung immer schärfer herausgearbeitet wurde. Der Exkursionsraum liegt südöstlich von Basel und umfaßt folgende Landschaften: 1 die Hochregion des Gempenplateaus, des westlichsten Teiles des Tafeljuras, mit Rodungsdörfern teils sehr alter Entstehung und in jüngern Ausbauphasen entstandenen Einzelhöfen; 2 die angrenzenden Räume des unteren Birs- und Hochrheintales mit ihren quartären, altbesiedelten Terrassenfluren, Industriesiedlungen und belebten Verkehrswegen im vorörtlichen Bereiche Basels; 3 die Übergangszone zwischen den Räumen 1 und 2. Birstal und Gempenplateau werden getrennt durch die vom Grenzacherhorn südwärts ziehende Rheintalflexur. Der Landschaftsstil dieser Übergangszone wird durch die in Anlehnung an die westwärts einfallenden mesozoischen Schichten entstandenen Hangfluchten, Schenkelberge und Kombentälchen geprägt. Im Gegensatz dazu sind die nördlichen und östlichen Flanken des Plateaus als Erosions- und Denudationsformen innerhalb des Tafeljurabaues angelegt worden.

\section{TECHNISCHE ANGABEN}

\section{Verbindungen und Unterkunft}

Alle Talsiedlungen sind mit der $S B B$ zu erreichen; Vorortstrambahnen von Basel nach Arlesheim, Dornachbrugg, Muttenz, Pratteln. Tägliche Postautokurse morgens, mittags, abends in beiden Richtungen auf den Routen Dornach-Gempen-Hochwald, Grellingen-Seewen, Liestal -Büren, Liestal-Nuglar. Übernachtungsmöglichkeiten finden sich in allen Orten am Fuße des Gempenplateaus sowie bei vorheriger Anmeldung in Gempen und Hochwald selbst.

$? 2$ Karten und Literatur

1 Karten

Landeskarten. Basel (1047), Arlesheim (1067), Paßwang (1087).

Geologische Karten: Gutzwiller, A. und Greppin, E. (1916) : Geologische Karte von Basel,

1. Teil Gempenplateau und unteres Birstal, Spezialkarte 77.

Herzog, P. (1956): Geologische Karte des Tafeljuras südöstlich von Basel, in 26.

Koch, R., Lehner, E., Waibel, A., Mühlberg, M. (1936): Geologischer Atlas der Schweiz, 99, Laufen-Mümliswil. 


\section{Literatur}

Aus Raumgründen hier ausgelassen. Wichtig und öfters zitiert sind:

2 Annaheim, H. (1961/62): Zur Geographie des Grundbesitzes der Christoph Merian'schen Stiftung. Regio Basiliensis III/1, p. 5- H+.

9 Boss, H. (1881): Urkundenbuch der Landschaft Basel, Basel.

13 Burckhardt, G. (1925): Basler Heimatkunde, Bd. I, Basel.

18 Eglin, J. (1958): Die historische Grenzsteinsammlung auf dem Kirchhof zu Muttenz, Muttenz.

2o Frohnmeyer, O. (1917): Gempenplateau und unteres Birstal, Diss., Basel.

23 Geschichte der Landschaft Basel und des Kantons Basellandschaft (1932), 2 Bde., Liestal.

25 Herzog, P. (1956): Die Tektonik des Tafeljuras und der Rheintalflexur südöstlich von Basel. Eclog. geol. Helv. 49, 2, p. 317-362.

36 Muggli, H. W. (1960): Arlesheim und seine Landschaft. Jurablätter.

39 Muttenz (Planung): Regio Basiliensis (1958/59), Bd. I, p. to. Regio Basiliensis (1961/62), Bd. III, p. 278-28o.

56 Wittmann, O. (1961): Die Niederterrassenfelder im Umkreis von Basel und ihre kartographische Darstellung. Basler Beiträge zur Geographie und Ethnologie, H. 3.

58 Zeugin, E. (1954): Pratteln (Beiträge zur Kulturgeschichte eines Bauerndorfes 1525-190o). Pratteln.

\section{EXKURSIONSROUTEN (siehe Abb. 1)}

\section{1 Übersicht der Routen}

Hauptroute I: ganztägige Fußwanderung.

Dornachbrugg, 29+ $\mathrm{m}$ (Trambahn Basel - Dornachbrugg, SBB-Station Dornach - Arlesheim) - Arlesheim, $335 \mathrm{~m}$ - Ränggersmatt - Schönmatt, $591 \mathrm{~m}$ - Stollen - Schartenflue, $759 \mathrm{~m}$ - Gempen, $676 \mathrm{~m}$ - Hochwald, 620 m - Herrenmatt - P. $576 \mathrm{~s}$ Herrenmatt — hinunter bis zum Fluhabfall w P. 616 (Hutzmeflue) — zurück nach P. 576 - Trockental ö P. 576 - Ziegelschüren - Bödeli w Seewen, $5+4 \mathrm{~m}$ - Fulnau - zurück auf Autostraße - Seetel - Grellingen (Station SBB); vom Bödeli eventuell mit Postauto nach Grellingen.

Weglänge $21 \mathrm{~km}$, ca. $61 \frac{1}{2}$ Stunden.

Varianten (Teilstrecken) zu Route I:

Ia Muttenz, $291 \mathrm{~m}$ (Trambahn- und SBB-Station) - Wartenberg, $479 \mathrm{~m}$ - Egglisgraben - Schauenburgflue, $663 \mathrm{~m}$ - Stollen, weiter nach R. I.

Ib Pratteln, $296 \mathrm{~m}$ (Trambahn- und SBB-Station) - Ebnet - Neu Schauenburg, 48o m Schauenburgflue, $663 \mathrm{~m}$ - Stollen, weiter nach R. I.

Ic bis P. 576 s Herrenmatt nach R. I, dann Falkenflue, $62+\mathrm{m}$ - Luegi - Duggingen, $331 \mathrm{~m}$ (SBB-Haltestelle).

Hauptroute II: ganztägig mit Benützung eines PW

Arlesheim, $335 \mathrm{~m}$ - Dornachbrugg, $294 \mathrm{~m}$ - Dornach — Schloß Dorneck - Dornach Gempen, 676 m, zu Fuß auf Schartenflue, 759 m zurück nach Gempen — Hochwald, von hier zu Fuß über Herrenmatt - P. 576 s Herrenmatt, hinunter bis zum Fluhabfall w P. 616 (Hutzmeflue) - zurück nach P. 576 - Trockental ö davon bis P. 602 - P. 616 Straße s Hochwald Hochwald - weiter mit Auto: Bödeli - Bergsturzriegel, zu Fuß nach Fulnau und zurück zur Straße - Seewen, $54+\mathrm{m}$ - Büren, $440 \mathrm{~m}$ - St. Pantaleon - Nuglar, $487 \mathrm{~m}$ - Oristal — Liestal, $315 \mathrm{~m}$; eventuell auf schmalem Sträßchen von Nuglar über Sichtern direkt nach Liestal.

Weglänge Fahrten: $30 \mathrm{~km}$; Wanderweg: $7 \mathrm{~km}$, Zeitbedarf ca. +—5 Stunden.

\section{Hauptroute I (Abb. 1)1}

1 Dornachbrugg $(294 \mathrm{~m})$. Um einen zentralen Platz, von dem vier Straßen ausstrahlen, reihen sich Gasthöfe, Ladengeschäfte, das Amtshaus des solothurnischen Bezirks Dorneck und das Kapuzinerkloster. Sie verraten, daß die in einer Ecke der Gemarkung Dornach erwachsene Siedlung in erster Linie zentralen Funktionen dient.

$s$ Der in der Achse der Birsbrücke liegende Ortskern wird von neuen Wohnquartieren eingerahmt, die im Zusammenhang mit der Industrieentwicklung entstanden sind. Die bäuerliche Siedlung der Gemeinde ist das auf der Hochterrasse gelegene Dornach («Oberdornach»). Dornachbrugg ist die einzige Altsiedlung im untern Birstal, welche unmittelbar am Birslauf auf der untern, von Überschwemmungen gefährdeten Stufe

1 Marginalien: R Relief, K Klima, G Gewässer, Bi Biosphäre, Be Bevölkerung, S Siedlung, W Wirtschaft, WL Landwirtschaft, WJ Industrie/Bergbau, V Verkehr, P Politische Geographie. Abkürzungen: E Osten, W Westen usw., ö östlich, w westlich usw., r., l. rechts, links. ON Ortsname, FN Flurname, Jh Jahrhundert, Gs Gaststätte. 


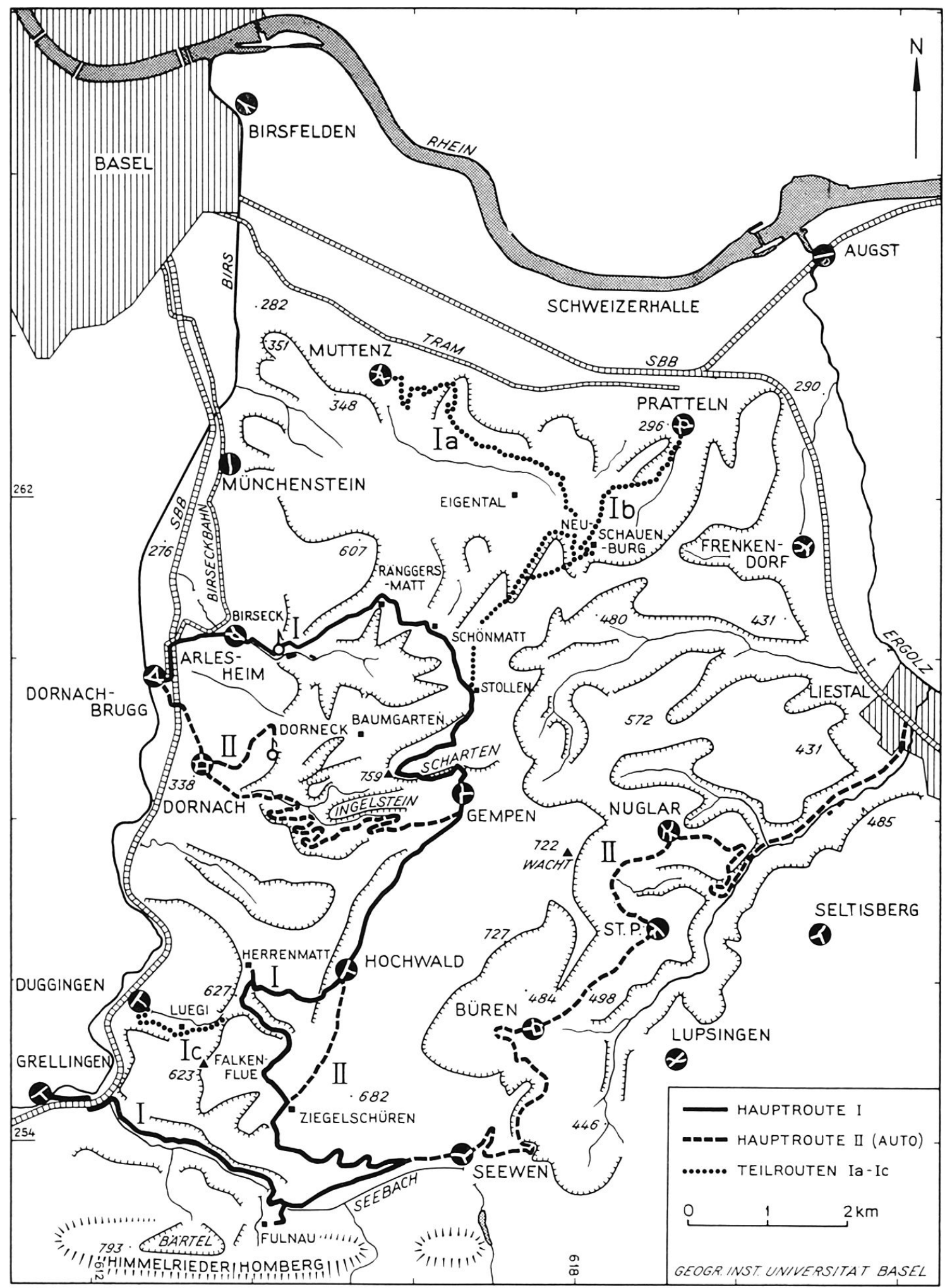

Abb. 1 Übersichts- und Routenkarte

der Niederterrasse entstanden ist. Entscheidende Entwicklungsimpulse vermittelte die Gestaltung des Birsbettes an dieser Stelle, bot sich doch nicht nur die Möglichkeit zum Brückenschlag, sondern auch zur Wasserkraftnutzung. Der Fluß hat sich in die leicht nordwärts fallenden Sandsteine der oligozänen «Molasse alsacienne» epigenetisch einge- 


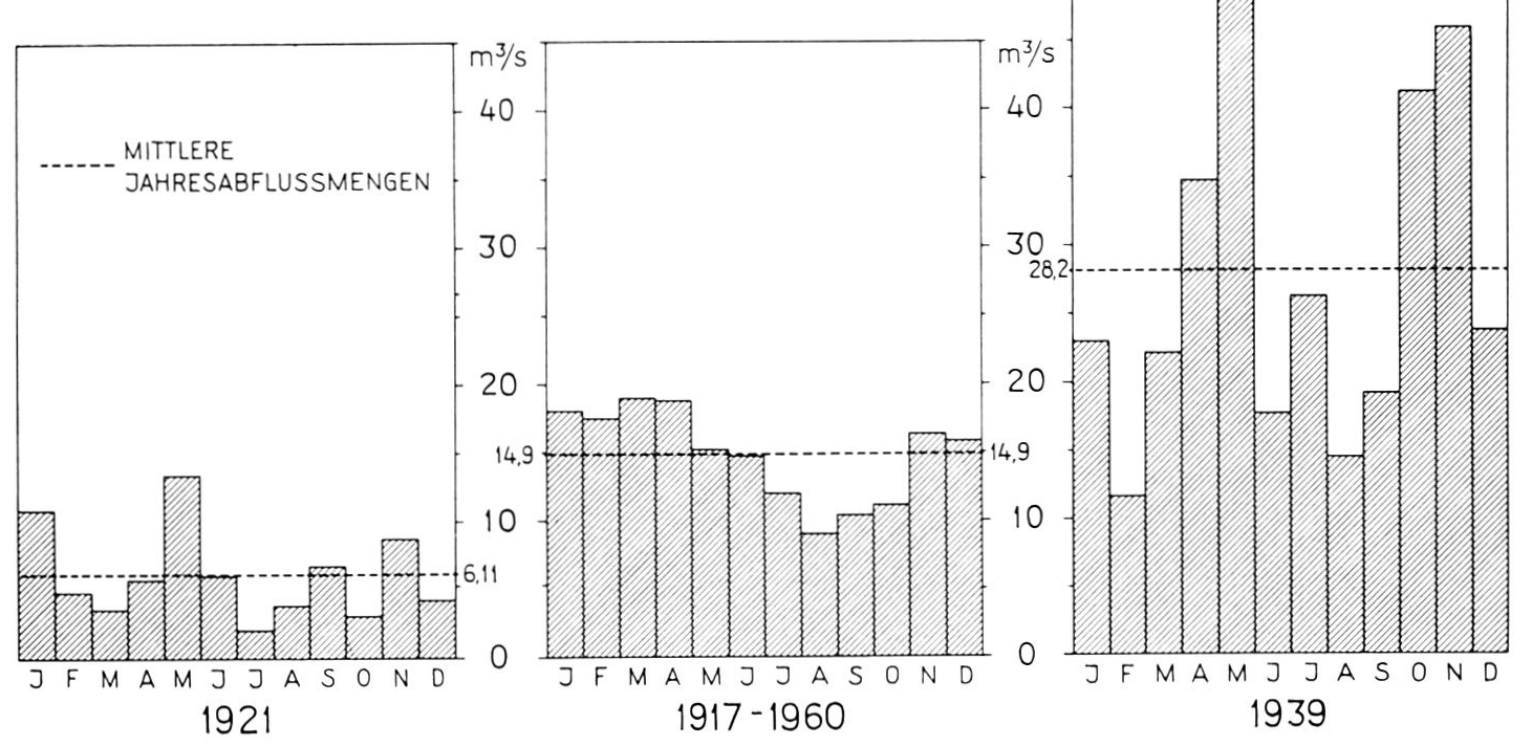

\section{Wasserstände 1960}

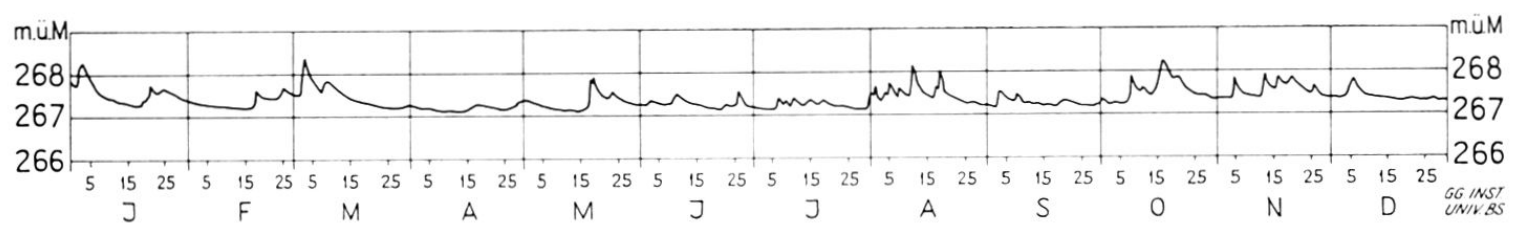

Abb. 2 Abflußverhältnisse der Birs bei Münchenstein: Mittlere monatliche Abflußmengen für das «Normaljahr» (Mittel 1917-1960), für ein extrem trockenes (1921) und extrem feuchtes (1939) Jahr. Die Kurve veranschaulicht den Wechsel der Wasserführung

schnitten und unterhalb einer heute von einem Stauwehr gekrönten Wasserfallstufe eine Schlucht eingenagt ${ }^{2}$.

Epigenesen der Birs haben auch an zwei weitern Stellen unterhalb von Dornachbrugg zum Brückenschlag eingeladen. So erleichterte der durch den Fluß angeschnittene Hauptrogenstein nördlich von Münchenstein die dreifache Überbrückung beim «Bruckgut» (Bahn, Trambahn, Straße, erstmals $1+35$ erwähnt, 12, VI, 31, 379). Die Stromschnellen im Keuper der Neuen Welt ermöglichten einen weitern Brückenschlag (1915 Straßenbrücke) und die Ableitung des St.-AlbanTeiches (Birswehr beim «Wasserhaus») eines der beiden alten Gewerbekanäle Basels. Die ersten Brücken bei St. Jakob waren vermutlich lediglich Fußgängerstege (1102/3 urkundlich «ad pontem Birse», 53, Bd. I, 14, 41), querten doch die Wagen die Birs bis ins 19. Jh in Furten; die StraBenbrücke entstand erst 1892 nach erfolgter Birskorrektion $1880-82(2 ; 11)$. Dagegen wurde die Brücke bei Birsfelden schon 1425 erbaut.

G Ober- und unterhalb der Epigenese pendelte der durch seine wiederholten Hochwasser gefürchtete Fluß (Abb.2) ursprünglich in der Talebene; oberhalb der Brücke wurde das linke Ufer vor einigen Jahren gesichert, während die Birs unterhalb der Schlucht nach jahrhunderte-

V langen Bemühungen 1865-75 endgültig korrigiert wurde, ein Werk, welches die Voraussetzungen zum Bau der Jurabahnlinie und zur Besiedlung der rechtsseitigen Talflächen bot (Weidenhof 1828 , s. 2). Unmittelbar oberhalb der Wasserfallstufe wurde die Brücke im festen Sandstein-

2 Die verschüttete tiefste Talrinne zieht zwischen Dornachbrugg und Reinach in der Talmitte dahin und scheint den heutigen Flußlauf mehrmals zu kreuzen (45). Der Grundwasserstrom dieser Rinne wird an verschiedenen Stellen zur Wasserversorgung angezapft (Reinach, Münchenstein). 


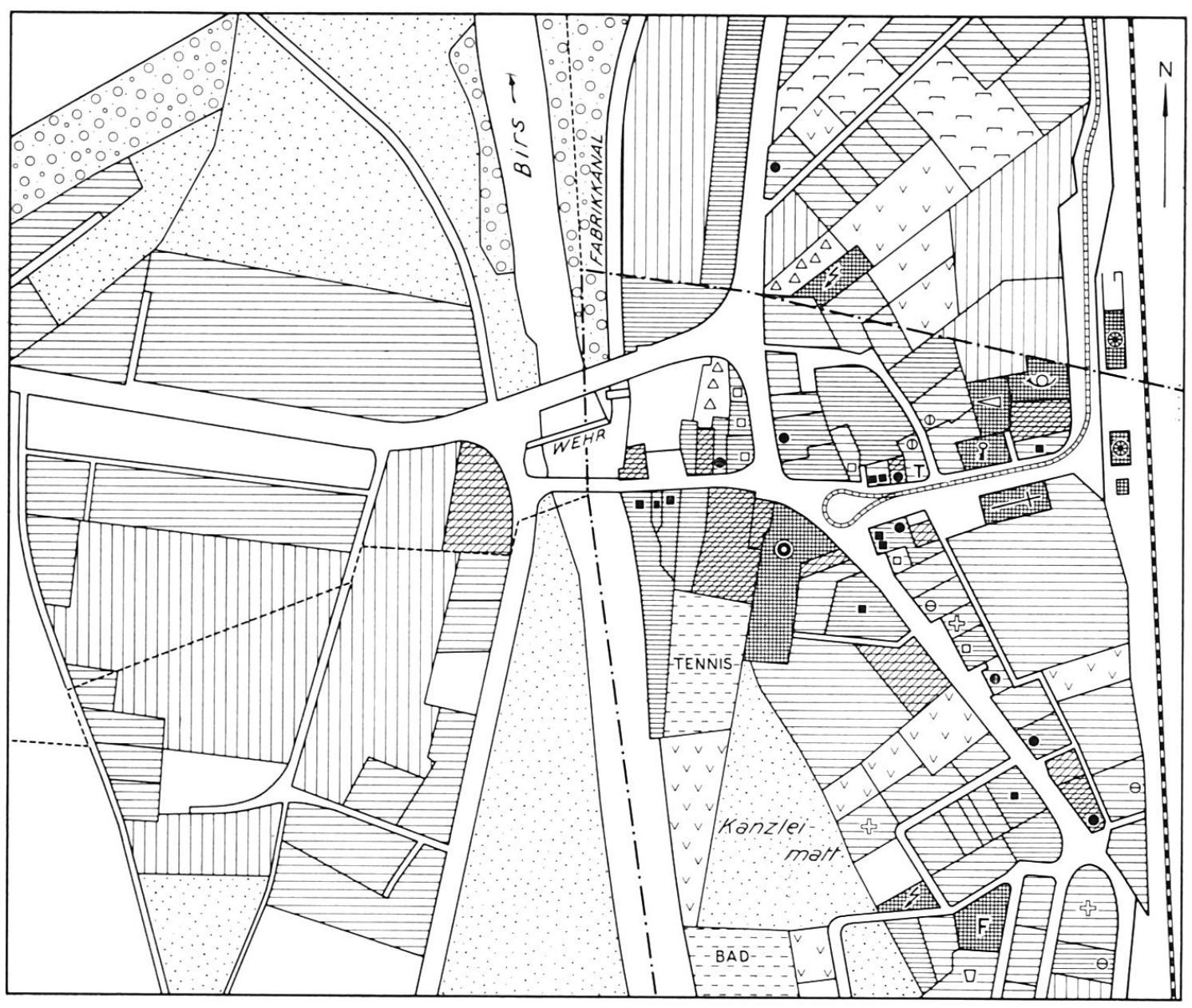

WOHNBAU, ZWEIGESCHOSSIG
WOHNBAU,
HOHER ALS ZWEIGESCHOSSIG
OEFFENTLICEE UND SOZIALE
DENSTE, GESCHAFTSBAU

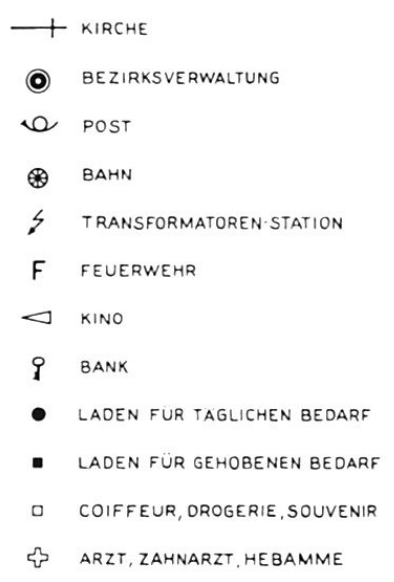

$\Theta$ WERKSTATT

(1) AutOrEPARATUR-WERKSTATt

T tankstelle

D GARTNEREI

EISENBAHN

TRAM

----- GEMEINDEGRENZE

-.- KANTONSGRENZE

$\underbrace{50 \quad 100 m}_{1}$

Abb. 3 Funktionalplan von Dornachbrugg

Widerlager der Ufer verankert. Als wohl ältester fester Birsübergang unterhalb Angenstein besaß sie zweifellos schon frühzeitig größere Verkehrsbedeutung $(20 ; 28)$ :

1485 erste Erwähnung der Brücke (46).

1493 erste Erwähnung des solothurnischen Brückenzolls.

1499 Abbruch der Brücke durch die Kaiserlichen beim Rückzug nach der Schlacht von Dornach (Denkmal beim Kapuzinerkloster, 1949 eingeweiht).

Mitte 17. Jh.: Holz-durch Steinbrücke ersetzt (Brückenheiliger Johannes von Nepomuk), Befestigung des Überganges durch Solothurn. 


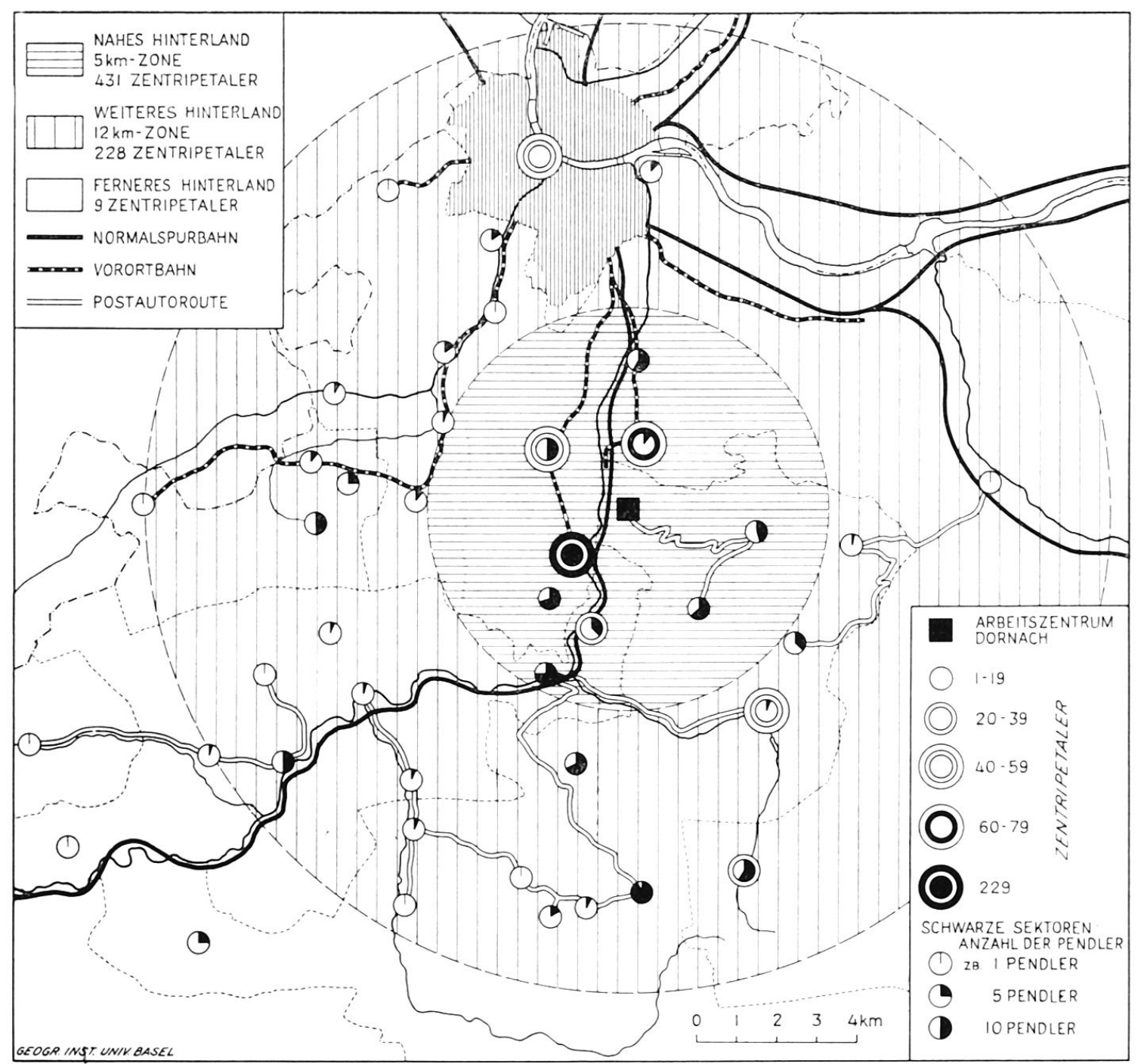

Abb. 4 Zentripetaler - Raum von Dornach (1941). Die Industrieansiedlung im Zentrum des Siedlungsdreiecks Arlesheim-Dornach-Reinach hat dazu geführt, daß Dornach in der Pendlerbewegung als Nebenzentrum zu Basel im Birseck erscheint und im Gegensatz zu den anderen Gemeinden einen Einpendlerüberschuß aufweist

1813 Brücke samt Brückentor von Birs weggerissen.

1823 Fertigstellung der heute noch bestehenden Brücke (Brückeninschrift).

1956/57 Erstellung der neuen Straßenbrücke $25 \mathrm{~m}$ flußabwärts; die alte Brücke dient nur noch dem Fußgängerverkehr.

Als Brückensiedlung in ausgezeichneter Verkehrssituation wurde Dornachbrugg Träger verschiedener zentraler Funktionen (Abb. 3 ).

1648 wird nach dem Umbau der alten Mühle zum auffallenden Treppengiebelhaus («Unteres Schlößli») hier die Amtsschreiberi des Bezirks Dorneck untergebracht und noch heute ist Dornachbrugg trotz der extrem peripheren Lage des Ortes innerhalb des Bezirks dessen Hauptort. 1672 läßt sich in diesem Vorposten solothurnisch-katholischen Landes der Kapuzinerorden nieder (Klosterbau 1676 vollendet). 1875 wird die Siedlung Station der Jurabahn nicht nur für Arlesheim und Dornach, sondern auch für das über die Brücke leicht erreichbare Reinach und 1902 endlich Endstation der Birseckbahn.

WJ Im Zuge der beginnenden Industriedezentralisierung faßt 1826 die Industrie von Basel aus hier Fuß (Schappespinnerei, gegr. 1824 in Basel, Lage der Fabrik an der Birs auf Arlesheimer Boden, heute ergänzt durch Wollspinnerei Sisi). Die Wasserfallstufe wird durch ein Stauwehr zur Ableitung eines Gewerbekanals gekrönt. 1895 erhält der Ort mit der Gründung der Metallwerke Dornach seine führende Industrie (etwa $1200 \mathrm{~m}$ oberhalb der Brücke an einem besonderen Ge- 


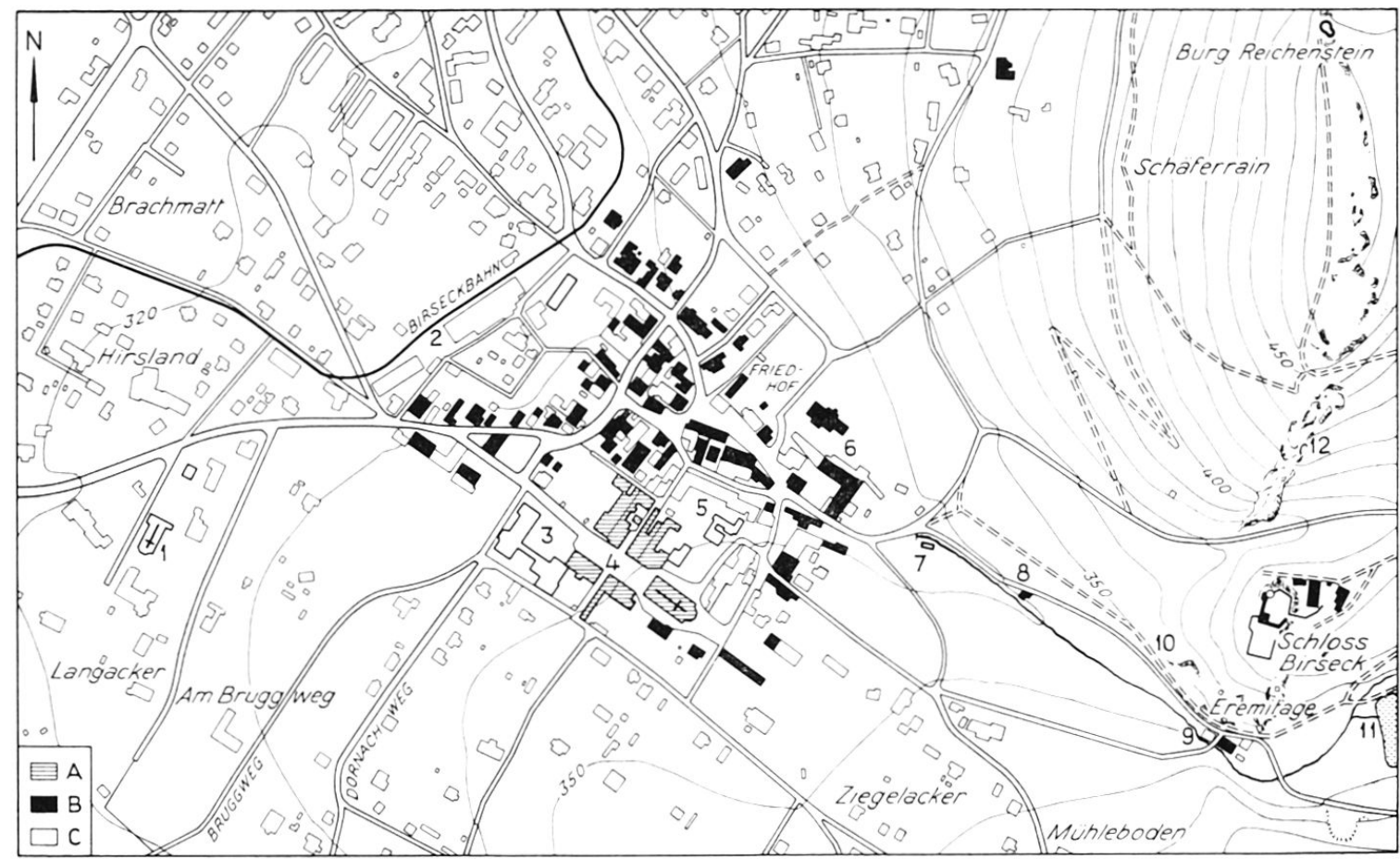

Abb. 5 Plan von Arlesheim. A ehemaliger Dombezirk, B Baubestand im Jahre 1896, C Zuwachs 1896-196o; 1 Reformierte Kirche, 2 Tramdepot, 3 Schule, 4 Domplatz, 5 Bezirksstatthalterei, 6 Andlauerhof, 7 Alemannengrab, 8 ehemalige Schleife, 9 ehemalige Mühle, 1o Halbhöhle «Rittiplatz», paläo- und neolithische Station, 11 Schloßweiher, 12 Hohlenfels, mesolithische Station

werbekanal)3. Mit anderen Gewerbebetrieben (Sägerei usw.) üben die beiden großen Industriebetriebe eine bedeutende Anziehungskraft auf die nähere und weitere Umgebung aus, so da $\beta$ Dornach in der Pendelwanderung einen nicht unerheblichen Zentripetalerüberschuß aufweist (1941: 668 Ein-, 307 Auspendler; 1950: 668 Ein-, 559 Auspendler; s. Abb. 4).

P Von der zentral und industriell bedeutenden Brückensiedlung aus wird das Siedlungsgefüge innerhalb des Siedlungsdreiecks Arlesheim - Dornach - Reinach immer dichter; es greift über die Kantonsmark zwischen Baselland und Solothurn und die Grenzscheiden der vier Gemeinden Dornach, Arlesheim, Reinach und Aesch hinweg, welche wohl kaum zufällig wenig unterhalb der Brücke an einem alten Grenzpunkt zusammentreffen.

2 Die Straße nach Arlesheim erreicht nach dem Übergang über die Bahn die obere

R Stufe der Niederterrasse (Akkumulationsniveau, Al nach 56) und kurz darauf die breite Verebnung der Hochterrasse (Nagelfluhbänke l. der Straße). Dann r. abzweigend am Schulhaus Arlesheim vorbei zum Domplatz.

\section{Arlesheim, $334 m$ (Abb. 5)}

S Die rechteckige, von einem Brunnen geschmückte Domplatzanlage mit den flankierenden ehemaligen Domherrenhäusern und der eindrucksvollen Domfront wurde in der zweiten Hälfte des 17. Jh geschaffen.

Die milde Landschaft, die Nähe Basels, mehr jedoch der allerdings schwache Schutz des Schlosses Birseck und der starke Schirm der Eidgenossenschaft veranlaßten den Bischof, das 1529 aus dem reformierten Basel nach Freiburg i. B. geflüchtete Domkapitel nach Arlesheim zu verlegen (1678). Innert vier Jahren wurde südlich des Dorfes auf einer Anhöhe die einheitliche hochbarocke Kapitelsiedlung erbaut. Der 1681 geweihte Dom wurde 100 Jahre später im Rokokostil fertiggestellt. Mit dem Übergang der fürstbischöflichen Länder an Frankreich (1793) wurde

3 1960: über 1100 Angestellte und Arbeiter, wovon um die 200 Pendler aus ca. 5o elsäßischen Gemeinden. 
das Domkapitel aufgehoben und der Dom ging in den Besitz der Gemeinde über. 1815 dem Kanton Basel zugesprochen, wurde Arlesheim Bezirkshauptort, eine Funktion, welche es auch nach der Teilung von Stadt und Landschaft (1833) beibehielt; damals wurden die neun katholischen Gemeinden aus dem bischöflichen Gebiet (Vogtei Birseck sowie Aesch und Pfeffingen von der Vogtei Pfeffingen) mit den 6 reformierten Gemeinden Muttenz, Münchenstein, Binningen, Bottmingen, Biel und Benken zum Bezirk Arlesheim zusammengeschlossen. Bezirksgericht, Statthalterei und Bezirksschreiberei hielten Einzug in die Domherrenhäuser.

S Vom Domplatz hinunter in den alten Dorfkern. Anders als die Domherrensiedlung schmiegt er sich nördlich des Domplatzes dem schwach eingesenkten Talboden des Dorfbaches an. Wenn auch die agrarischen Anwesen immer mehr zurücktreten, so ist doch die ursprüngliche Anlage als doppelte Bachzeile mit traufständigen, teilweise aneinandergebauten Häusern noch gut zu erkennen.

Der Ortsname - es handelt sich bei Arlesheim um den südlichsten «heim»-Ort der Region - und der Fund eines alemannischen Steinkistengrabes lassen vermuten, daß der Ort auf die alemannische Landnahmezeit zurückgeht.

Erste Erwähnung der Siedlung im Jahre 7o8; dem elsäßischen Kloster Hohenburg (heute Odilienberg bei Barr) übergibt Odilie, die Tochter des Herzogs Adalrich, den Dinghof A. (beim heutigen Andlauerhof?) (9, 1, 4). Die kleine, ehemals auf dem Friedhof stehende, 1625-29 neu erbaute und 1793 abgerissene Dorfkirche war der Hl. Odilie geweiht. 1239 wurde die Siedlung mitsamt den beiden Burgen Ober- (Reichenstein!) und Unter-Birseck vom Basler Bischof gekauft. Die Siedlung gehörte - vermutlich infolge der beschränkten Anbaufläche auf der Terrasse jahrhundertelang zu den bescheidensten Dörfern des Birsecks (1585: 31 Häuser, ca. 25o Ew.). Dies änderte sich erst mit dem 17. Jh. Als Verwaltungszentrum der Vogtei Birseck (A., Reinach, Oberwil, Therwil, Ettingen, Allschwil, Schönenbuch) und Sitz des Domkapitels erhielt Arlesheim bedeutende zentrale Funktionen. Damals erhob sich Arlesheim über die andern Siedlungen des

$S$ Birsecks und wurde allmählich «ein Dorf ohne Bauern», wie ein Fürstbischof sagte. Der Verlust des Domkapitels (1793), ein schwerer Schlag für die Siedlung, wurde jedoch im Laufe des 19.Jh durch die Übernahme bezirksörtlicher Funktionen, die Ansiedlung von Industrie und die Entwicklung zum bevorzugten Wohnort von Basler Geschlechtern mehr als ausgeglichen. 1826 gründete die Familie Alioth die Schappespinnerei an der Birs (s. S. 246). Diese bedeutendste Familie Arlesheims, in deren Besitz auch das Schloß Birseck und der Andlauerhof sind, gründete zudem 1894 die Fabrik elektrischer Maschinen bei Münchenstein (heute Brown, Boveri \& Co.). Neben den Fabrikanten ließen sich immer mehr andere begüterte Basler in Arlesheim nieder; die Siedlung wurde zum Villenvorort (viele klassizistische Landhäuser namentlich s Hirsland und sw des Domplatzes).

Um das Gepräge einer gehobenen Wohnsiedlung nicht zu verlieren, wehrt sich die Gemeinde gegen den Bau größerer Komplexe von Mehrfamilienhäusern im Weichbild

Be der Ortschaft (Lit. 22; 24; 29; 51). Die von Obstbäumen übersäten Sonnenhalden wurden von Einfamilienhäusern überbaut, deren Bewohner großenteils in der Basler Industrie tätig sind (1960: Auspendler nach Basel: $688=27,9 \%$ der berufstätigen Bevölkerung). Diese Entwicklung wurde ermöglicht und verstärkt durch den Bau der Birseckbahn (1903); dadurch wurde Arlesheim in die Vorortsentwicklung von Basel mit einbezogen. Dies äußert sich in der durch die Zuwanderung bedingten starken $\mathrm{Zu}$ nahme der Bevölkerungszahl, im Wandel der soziologischen Struktur zur AngestelltenWohnsiedlung und von einer katholischen zu einer vorwiegend reformierten Gemeinde (1960: 48\% Reformierte, 45\% Katholiken, Abb. 8, 9).

s Vom Dorfkern die Dorfstraße talauf. Die letzte Gebäudegruppe 1. ist der Andlauerhof (ehemals Flachsländerhof). Er stammt aus der gleichen Zeit wie der Bau des Domhofes, wurde 1762 von Landvogt Karl von Andlau anstelle des Schlosses Birseck als Sitz der Landvogtei bezogen und heißt seither Andlauerhof (Barockisierendes, geschweiftes Scheunendach aus dem Anfang des 19. Jh).

WJ Nun dem Bach entlang gegen den Schloßfelsen von Birseck. R. das alemannische Steinplattengrab aus dem Dorf; das kleine Häuschen am Bach, die sog. «Schleifi», ist eine ehemalige Diamantenschleiferei. Die Wasserkraft des kleinen Baches nutzten auBerdem die alte Mühle am Ausgang des Gobenmatt-Tälchens (ehemals bischöflicher Besitz, um 1850 stillgelegt) und die ehemalige Oele (Gewinnung von Nußöl, zuvor Hammerschmiede) im Tälchen bei den Fischweihern des Domkapitels. 

Hollenberges auf, deren Korallenkalk (Malm) mit ca. $30^{\circ}$ nach W einfällt. Ihr Fuß bezeichnet die geologische Grenze zwischen Tafeljura und Rheingraben, zu welchem die mesozoischen Schichten in Form einer Flexur abgebogen sind.

Die Bildung der Rheintalflexur hatte in unserem Gebiet im unteren Rupélien (Oligozän, Meeressand) eingesetzt, was durch die Diskordanz zwischen der Auflagerung des Meeressandes und dem Rauracien von $14^{\circ}$ belegt wird (26). Andrerseits beweist der östlichste bisher bekannte Aufschlu $\beta$ von Molasse alsacienne auf dem Gempenplateau beim Hof Ziegelschüren, daß im Chattien der Höhenunterschied zwischen dem Rheingraben und der Region des Gempenplateaus noch nicht bestand oder doch gering war. Die Küste des Rupélien-Meeres wird durch von Pholaden angebohrte Strandgerölle aus dem Malmkalk bezeugt; eine Fundstelle findet sich s des Baches am Waldrand bei Birseck (614200/26o 125).

Beim Ausgang des Gobenmatt-Tälchens, gegenüber der Mühle, betritt man durch eine Höhle (Felsentor) die Eremitage, einen englischen Garten am Schloßberg, ein einzigartiges, zu wenig bekanntes Kulturdenkmal des ausgehenden 18. Jh.

1762 begann Balbina von Andlau, die Gattin des bischöflichen Landvogts, zusammen mit dem Domherrn Heinrich von Ligerz die Anlage des Gartens. Rousseaus Naturverehrung und Gessners Idyllendichtung spiegeln sich in der romantischen Gestaltung mit ihrem Gewirr von Pfaden, hölzernen Stegen, Aussichtsterrassen, einsamen Hütten, der Klause eines Waldbruders und zahlreichen Inschriften. 1785 war die Anlage vollendet; sie wurde während der Revolutionszeit zerstört, nachher aber wieder aufgebaut $(49)$. Einen besonderen Reiz bilden die zahlreichen Karsterscheinungen wie Höhlen und Halbhöhlen, welche durch ihre steinzeitlichen Funde eine frühe Besiedlung der Region belegen. Die Balm, die etwa $11 \mathrm{~m}$ über dem Bach beim «Rittiplatz» liegt, ergab Funde aus dem späten Magdalénien und aus der Mittelsteinzeit (Azilien, bemalte Kiesel: Totemgegenstände?) (t1). Weitere vorgeschichtliche Siedlungsplätze wurden in den Halbhöhlen am Hollenberg (s Birseck) und am Hohlen Felsen (n Birseck) gefunden.

$\mathrm{R} \quad$ Der Weg durch die Eremitage führt auf die Höhe des Schloßberges, der vom Schenkelberg des Hohlen Felsens durch ein kurzes Trockentälchen (ehemaliger Abfluß des Kombentales von Reichenstein?) getrennt ist. Von der einstigen stolzen Anlage des Schlosses Birseck stehen nur noch der Bergfried und die Kapelle (Zerstörung 1793). Neben der Ruine der Pächterhof.

S Die Schlösser Birseck (Unterbirseck) und Reichenstein sind vom Kloster Hohenburg zum Schutze von Arlesheim namentlich gegen die Froburger angelegt worden. 1239 wurde der Klosterbesitz an den Bischof von Basel verkauft, der die Burg Oberbirseck dem Geschlecht der Reich zu Lehen gab; die fortan Reichenstein genannte Feste zerfiel bald, wurde 1933 von einem Basler Industriellen erworben und zum Sommersitz ausgebaut. Birseck war bis 1762 Sitz des bischöflichen Vogtes, ging in der Revolutionszeit in Flammen auf und wurde durch die heutigen Besitzer (Familie Alioth) vor dem gänzlichen Zerfall bewahrt $(34,37)$. Die beiden Anlagen gehören zur Reihe von ehemaligen Höhenburgen und Wasserschlössern, welche in der näheren Umgebung Basels in so großer Zahl erbaut wurden, daß man geradezu von einer «Burgenlandschaft» zu sprechen geneigt ist, wie sie in dieser Art in der Schweiz wohl nur noch einmal, im Domleschg, auftritt. Sie sind bezeichnende Dokumente der Basler Kulturlandschaft des hohen Mittelalters und bezeugen die Erfolge des niedern Adels in einer Region geringer landesherrlicher Macht (33).

Überblick von der Aussichtsterrasse des Turmes: Im W der östlichste Teil der Sundgauer Tertiärplatte, das Bruderholzplateau, dessen oligozäner Unterbau mit quartären Schottern und Löß verbrämt ist. Im S die Landskron- und dahinter die mächtige Blauenkette, Falten des Kettenjuras, welche weit nordwärts in den Rheingraben vorstoßen. In der Tiefe die Terrassenlandschaft des Birstales; auf den altbesiedelten höhern Terrassen die bäuerlichen Siedlungskerne und eine weit ausgreifende vorörtliche Wohnüberbauung; darunter die erst nach 1800 von der Industrie und neuen Wohnquartieren besetzte Niederungszone. Physiognomisch besonders auffallend ist die lockere Hangbesiedlung um den grauen Koloß des Goetheanums auf einer zwischen der Ruine Dorneck und dem Hollenberg niedergegangenen Bergsturzmasse. Die Anthroposophen erhielten um 1910 vom Kt. Solothurn die Erlaubnis zur Niederlassung, und in der Folge siedelten sie sich an dieser günstigen, nahe der Grenze und einer Universitätsstadt gelegenen Stelle in großer Zahl an (47).

$\mathrm{R} \quad \mathrm{Im}$ E steigen in stärkstem Kontrast zur Kulturlandschaft des Birstales die waldreichen, nur da und dort von Rodungsinseln durchsetzten Hänge des Gempenplateaus an, welche sich im Dornachberg in mächtiger, geschlossener Flanke zur Höhe auf- 
schwingen, während sie nördlich davon in eine Reihe von Schenkelbergen aufgelöst sind. Hinter diesen folgen zunächst Ausräumungszonen im Oxfordien (Kombentälchen), aus denen weiter im E die meist dichten Wald tragenden Hauptrogensteinflanken emporragen.

5 Vom Schloß Birseck weiter auf dem Fahrweg nach Ränggersmatt (30 Min.). Gleich links ein Aufschluß in den gut gebankten Kalken des obern Doggers (Hauptrogenstein). An das Auftreten dieses Gesteins und des Korallenkalks sind die Härtekanten und Stufenränder des Gempenplateaus gebunden (Abb. 6). So wird der Übergang von den Plateauflächen von Baumgarten und Schönmatt von den Stufenkanten im Obern Hauptrogenstein gebildet; noch ausgeprägter neigt der Korallenkalk zur Fluhbildung (Scharten-, Falkenflue, Ingelstein). Die untern Stufenfronten im Doggerkalk sind von mächtigen, vermutlich periglazial-pleistozän gebildeten Schuttmänteln verhüllt (z. B. Mergelgrube ESE der Oeli im Gobenmatt-Tälchen).

Die Bildung der relativ geräumigen, handförmig gelappten Ausräumung der Gobenmatt ist durch die intensive Bruchbildung in der Flexurzone begünstigt worden. So führt vom Trockentälchen zwischen Birseck und dem Hohlenfelsen ein Bruch in die Gobenmatt hinein (26, Karte, Profile); quer zu ihm verlaufen zahlreiche Verwerfungslinien, die sich z. T. wohl nicht an den Gehängen morphologisch abzeichnen, denen aber mit einer Ausnahme die vier seitlichen Ausstülpungen der Ausräumung folgen. Die starke Schollenzerstückelung mag ganz allgemein den Eingriff der abtragenden Kräfte und damit die bedeutende Ausräumung erleichtert haben.

6 Die Route führt nun durch ein ebenfalls von einer Bruchlinie flankiertes 'Tälchen (NW: Hauptrogensteinstufe, SE: Flächen in Varianschichten und Callovien) hinauf zur Ränggersmatt, einer jungen Rodungssiedlung (ursprünglich Heuscheune, Anfang des 19. Jh zum Hof ausgebaut). Auf dem Fahrweg erreicht man in 10 Minuten die Schönmatt (590 m, Gs) und damit die Höhe des Gempenplateaus.

P Kurz vor der Schönmatt l. am Waldrand das Scheidflühli, eine alte natürliche Grenzmarke, die noch heute die Kantone BL und SO scheidet. Im Hochland der Gempentafel stößt der Kanton Solothurn am weitesten nordwärts vor, wobei die Grenze häufig dem Plateaurand folgt. Im W greift er über den Dornachberg bis ins Rheingrabenland hinunter; im E umfaßt er den ganzen Plateauhang bis zur Tiefe des Oristales. Alle Besitzungen im Raume des Gempenplateaus hat Solothurn durch Kauf von verschiedenen Herren in den Jahren 1485-1522 erworben, wobei es im Bestreben, sein Gebiet bis an den Rhein auszudehnen, in scharfem Konkurrenzkampf mit Basel stand, welches sowohl am Blauen (Amt Rotberg) als am Gempen das Nachsehen hatte, aber immerhin Muttenz und Pratteln entgegen den Bemühungen Solothurns erwerben konnte (s. S. 261).

Bi Die weite Verbreitung von Kalkböden, welche lediglich im S des Plateaus stellenweise von Verwitterungslehm bedeckt sind, macht es verständlich, daß der Wald nicht nur weithin die Hänge des Plateaus als fast geschlossener Gürtel überkleidet, sondern auch auf der Plateaufläche selbst auf umfangreichen Arealen stockt. Der durch die zahlreichen Verwerfungen bedingte häufige petrographische Wechsel spiegelt sich oft überraschend in der Waldvegetation (35). So findet sich am Chilchholz ( $\mathrm{n}$ Gobenmatt) auf Hauptrogenstein Flaumeichenbuschwald, während die mergeligen Böden von Steinsamen-Traubeneichenwald bestockt sind. Auf Callovien und anderen Mergeln und Tonen setzt sich stets der Eichen-Hainbuchwald, die Klimaxgesellschaft der collinen Stufe, durch, auf Hauptrogenstein dagegen die Klimaxvegetation der Submontanstufe, der Seggen-Buchenwald. Ausdruck des ozeanischen Klimacharakters ist das Vorkommen von Eiben ('Taxus baccata) und der etwas selteneren Stechpalme (Ilex aquifolium).

S Der am alten Baselweg (Gempen - Hof Gruth - Münchenstein) liegende Hof Schönmatt gehört zu der ältern Generation der Plateauhöfe.

Er erscheint schon in der Mitte des 17. Jh auf Plänen $(21,52)$. Noch älter sind die Höfe Unter-Gruth (1532, vermutlich jedoch schon um 1300 bestehend, $2 ; 27)$, Ober-Gruth $(1712 ; 2 ; 27)$ und der schon zur Zeit der Schlacht von Dornach (1499) erwähnte Hof Baumgarten. Erst nach der Aufhebung des Flurzwanges zu Beginn des 19. Jh entstanden Ränggersmatt, Stollen, Schar- 
tenhof, Chilchenrain ob Hochwald und Ziegelschüren (21). Der Großteil dieser Höfe liegt im nordwestlichen Teil des Plateaus weit abseits der beiden Tafeldörfer.

S Weiter nach Stollen $(638 \mathrm{~m})$, einer Weilersiedlung, welche zu Anfang des 19. Jh WL mit der Allmendteilung erwuchs $(21 ; 52)$; vorher bildeten deren Felder eine Zelge der Gempener Flur. Die Siedlung verfügt über eine relativ günstige Wasserversorgung, liegt sie doch nahe einer Bruchlinie, an welcher der Quellhorizont des Oxford ausgeht (ehemals 3 Sodbrunnen; gefaßte Quellen); sw der Höfe zwei Dolinen. Vom Weg Stollen - Gempen biegt die Route nach W ab und führt durch den Schartenwald zur Schartenflue (759 m Gempenfluh, Aussichtsturm, Gs). Aus flachliegendem, von der Verwitterung «zerschartetem»Korallenkalk aufgebaut, ist sie einer der Felsabstürze, welche die Plateauränder immer wieder akzentuieren und dank ihrer vorgeschobenen Lage ausgezeichnete Aussichtspunkte sind.

S Deshalb waren ehemals auf der Scharten- und Schauenburgfluh sowie auf der Anhöhe ösö Gempen (Wacht, 722.7) Hochwachten eingerichtet, deren östlicher Anschlußpunkt die Wacht auf der Sissacherfluh war; die Karte von D. Bruckner (1766) verzeichnet die Hochwacht der Schartenflue samt Wächterhaus.

Der Rundblick schweift bei klarem Wetter bis zu den Alpen, den Vogesen und dem Hochschwarzwald (Feldberg). Im W die vom Abtrag aufgeschlitzte Hochlandflanke; im Doggerkalk herauspräparierte Flächen (Höfe Schartenmatt-Baumgarten), dann im Oxfordien angelegte Ausräumungen (Ramstel und nördlich davon abzweigendes Kombentälchen) und Korallenkalkrippe Dorneck - Hollenberg, deren Mitte durch den Ausbruch des Bergsturzes (Goetheanum-Hügel) erniedrigt ist. Jenseits des Birstales das wellige Sundgauerhügelland, das im S durch die Pfirterkette (Hintergrund) und den Blauen überhöht wird; sö der Birsklus von Angenstein steigt als nördliche Randkette des Faltenjuras der Himmelrieder Homberg an. - Unterhalb der Hafenanlagen Basels die Oberrheinische Tiefebene mit Rhein, Rheinseitenkanal und Kembser Werk; ö davon das Markgräfler Hügelland mit dem nach W vorspringenden Isteiner Klotz (Malmkalksteinbruch). Im S ist dem Schwarzwald der Dinkelberg vorgelagert, der transrhenanische Teil des Tafeljuras. Zwischen ihm und dem zerschnittenen Nordhang des Gempenplateaus mit dem nordwärts vorspringenden Wartenberg das Hochrheintal. Ostwärts Blick über die Plateaus des Tafeljuras, aus denen im $\mathrm{S}$ die Ketten des Faltenjuras mit steilen Überschiebungsfronten aufsteigen; im SE die mächtige Antiklinale des Paßwangs $(1204 \mathrm{~m})$.

R Aufschlußreich ist der Blick auf die Plateauhochflächen im NE und SE (Abb. 6, 7). Wie die übrigen Teile des Tafeljuras ist auch die Gempentafel von zahlreichen Brüchen durchsetzt, welche vorwiegend in NNE-SSW-Richtung streichen; in Analogie zu den Brüchen im östlichen Basler Tafeljura darf man für diese Verwerfungen auf vormiozänes Alter schließen $(26,345 \mathrm{ff}$.).

Das durch die Bruchbildung entstandene Mosaik von Hoch- und Tiefschollen wurde vom nacholigozänen Abtrag weitgehend eingeebnet, so daß sich die Bruchlinien auf der heutigen Landoberfläche lediglich durch die Kontakte verschiedener Gesteinshorizonte oder durch an sie gebundene lokale Formkomplexe morphologisch auswirken. In Analogie zum östlichen Tafeljura dürfte die in 620-730 m Höhe liegende Pencplain mittelmiozän oder unmittelbar prämittelmiozän sein. 4

R Der Scharten dokumentiert aufs schönste, wie weitgehend der Abtrag die tektonische Oberfläche umzugestalten vermochte. Seinem Hangfuß entlang verläuft eine vom Ramstel heraufziehende Bruchlinie, welche gegen die Schauenburgflue abbiegt. Sie trennt den bis auf den obern Dogger abgetragenen Horst von Baumgarten-Horn von dem tektonisch ca. $80 \mathrm{~m}$ tiefer, orographisch jedoch ca. $150 \mathrm{~m}$ höher liegenden Scharten. Es liegt sonach ein eindrücklicher Fall von Reliefumkehr vor.

Die nördliche Hangstufe des Scharten ist anlagemäßig wohl eine Bruchlinienstufe; da jedoch der Fuß der Korallenkalkstufe aus den Mergeln des Oxford und obern Doggers der Landterrasse von Baumgarten gebildet wird, besitzt sie alle Kennzeichen einer Schichtstufe (1o). Noch deutlicher ist der Schichtstufencharakter der Scharten-Südflanke. Längs einer WE streichenden, das Dorf Gempen kreuzenden Verwerfung, welche orographisch nur schwach in Erscheinung tritt, ist die Scholle südlich des Schartens um ca.90 m abgesunken. Ursprünglich als Bruchstufe ange-

4 Die Hochflächen des Gempenplateaus sind - abgesehen von einzelnen Abschnitten - sowenig wie das höchste Plateau des übrigen Tafeljuras «Tafeln»im streng morphologischen Sinne, sondern die Strukturen schneidende Peneplainformen. Im Interesse objektiver Forschung zmpfiehlt es sich, diesen Begriff rein morphographisch zu fassen und nicht mit vielleicht problenatischen genetischen Aussagen zu belasten. 


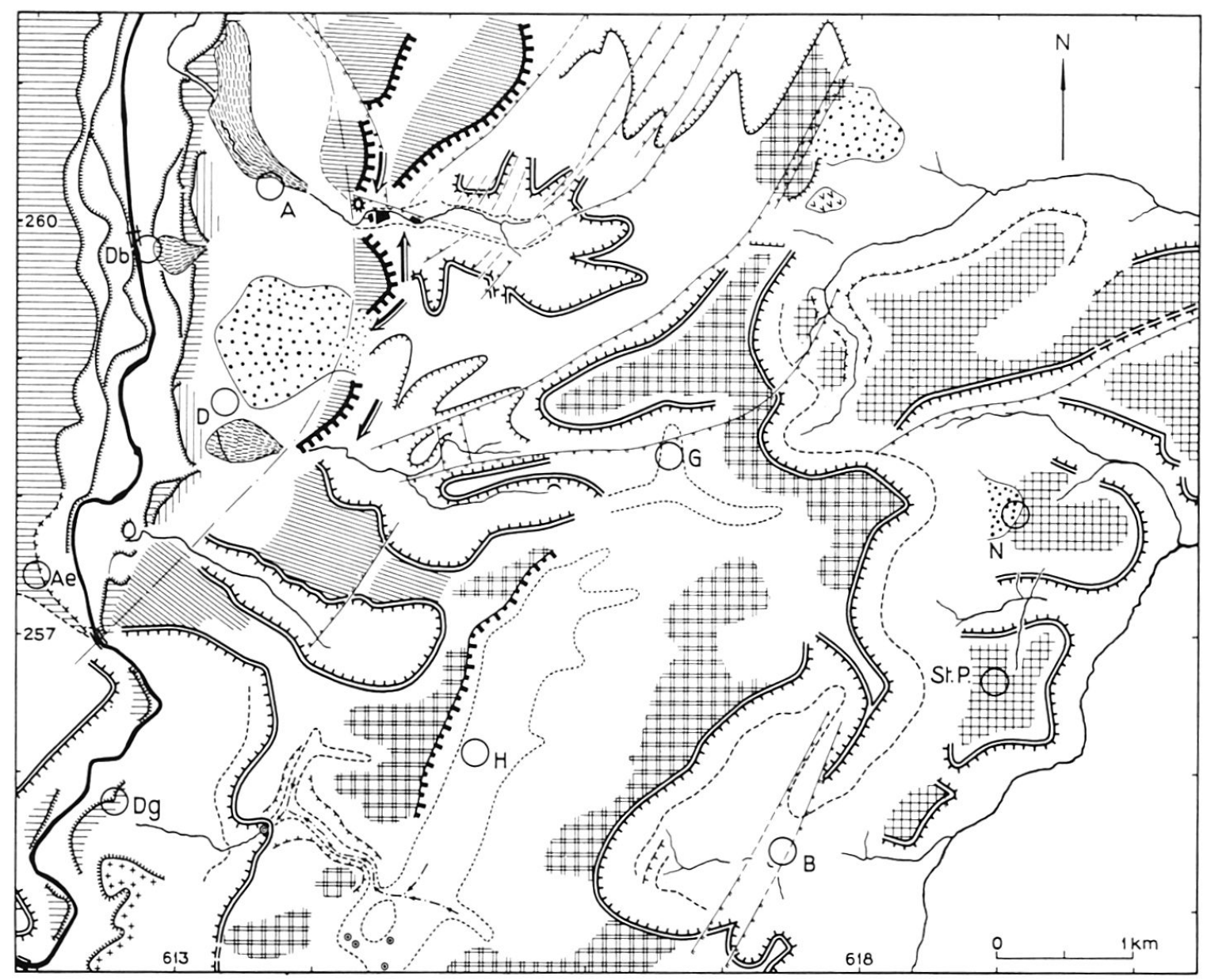

1 Abrragsformen

2 Akkumulationsformen

RESTE ALTER VEREBNUNGEN

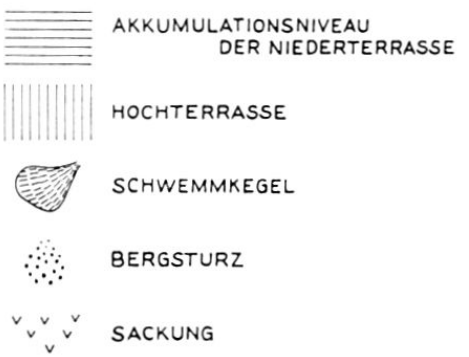

ПT्तTT HARTESTUFE,SCHICHTSTUFE

IIIm SCHICHTRIPPE

MTI BRUCHSTUFE

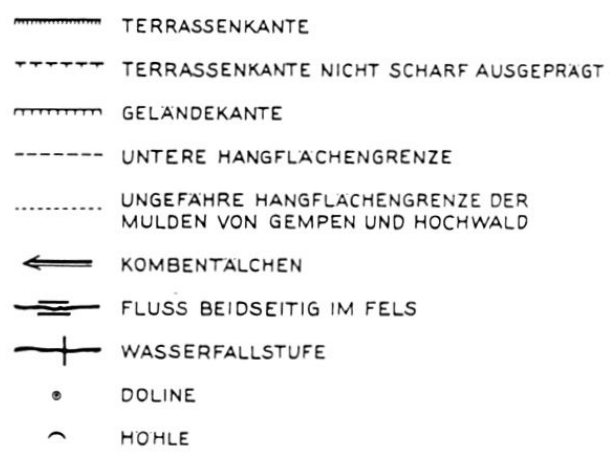

3 Gewasser

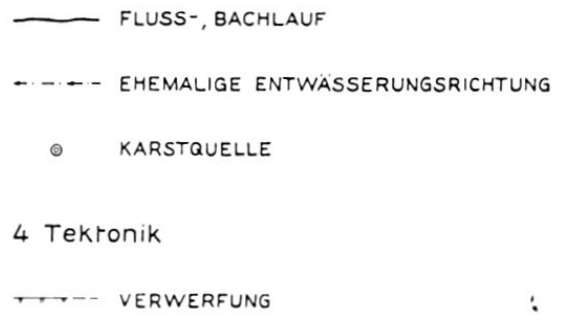

ᄀ HOHLE

Abb. 6 Geomorphologische Skizze des Gempenplateaus 


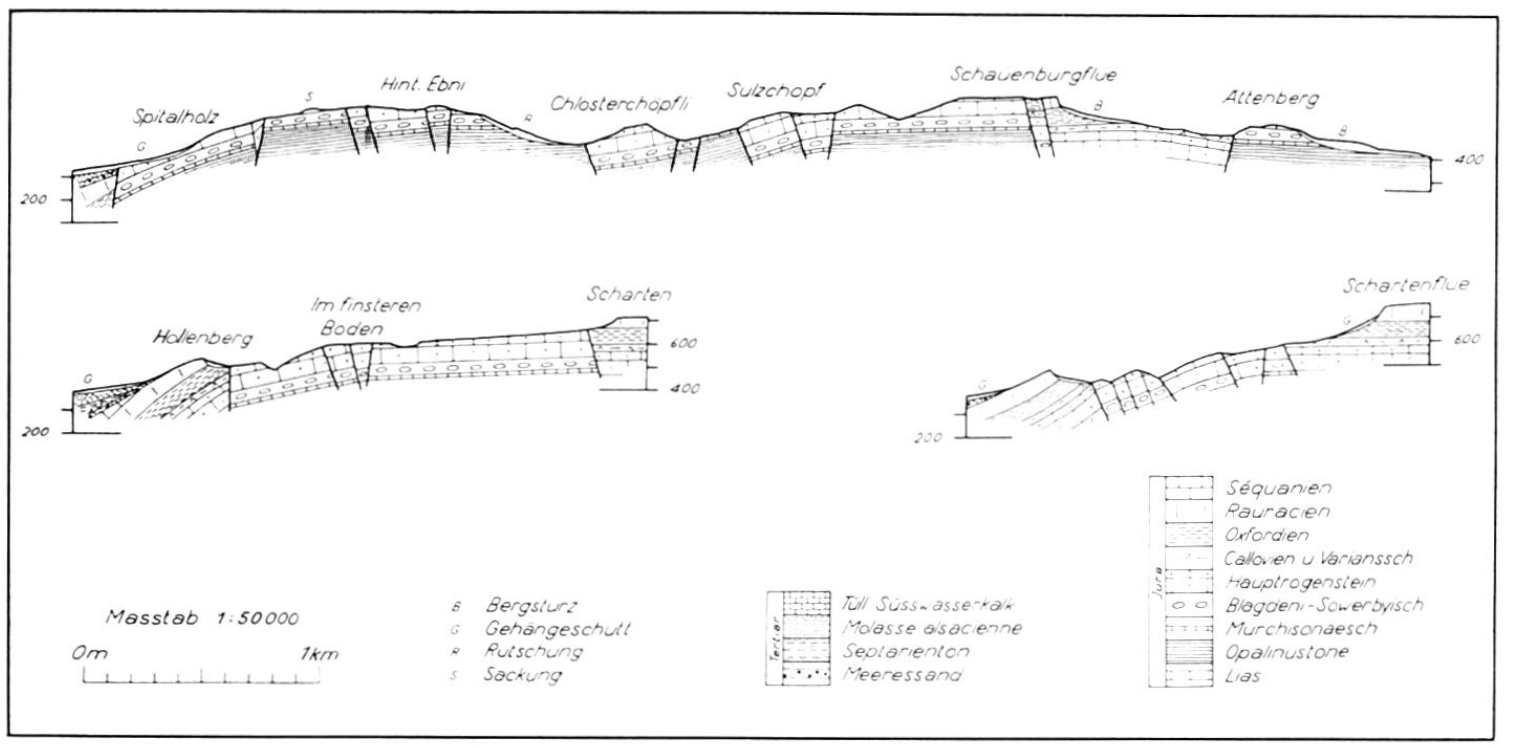

Abb. 7 Geologisch-tektonische Profile durch die Gempentafel, nach P. Herzog

legt, ist jedoch die Flanke über dem Oxfordmergel um 120-20o m zurückverlegt worden und bildet daher in ihrer heutigen Form wie die Schartenflue selbst ebenfalls eine Schichtstufe 5. Der Scharten als Ganzes ist als Härtling (Monadnock) in der Peneplain aufzufassen, und dies um so mehr, als seine nordöstliche Flanke allmählich in den zwei Verwerfungen kappenden Peneplainabschnitt von Gmeinacher-Horn übergeht; auch die heute morphologisch inaktive Talmulde von Gempen greift über den Bruch von Gempen hinweg auf die karstholden Gesteine des Schartens über 6. - Aus der Tiefscholle südlich des Schartens wurde der Korallenkalksporn des Ingelsteins herauspräpariert (Glitzersteinhöhle, S. 262).

7 Nun nach Gempen (s. unten), dann weiter auf der Fahrstraße nach Hochwald; sie quert zunächst ein kleines Muldental, welches auf den Plateaueinschnitt gerichtet

$\mathrm{R}$ ist, der ins Ramstel hinunterführt, dann durch den Grenzwaldgürtel zwischen den Gemarkungen der beiden Hochlanddörfer nach Hochwald. Die sich südwärts senkende Mulde von Hochwald ist asymmetrisch gebaut. Im W wird sie von einer steilen, um die $50 \mathrm{~m}$ hohe Bruchstufe flankiert, deren Höhe bis auf den Korallenkalk abgetragen ist; im E senken sich dagegen die aus leicht westlich geneigtem Sequenkalk bestehenden Verflachungen allmählich gegen die Talachse ab und sind hier von Verwitterungslehm bedeckt.

s Gempen $(680 \mathrm{~m})$ und Hochwald $(620 \mathrm{~m})$, die beiden einzigen Dorfsiedlungen der Plateauhöhe, liegen beide in relativ windgeschützten Mulden innerhalb traditioneller Gewannfluren, welche von einem nahezu geschlossenen Waldgürtel eingefaßt sind.

Beide kamen, Gempen aus der Hand der Basler Familie von Efringen, Hochwald aus jener des Basler Bischofs, um 1500 durch Kauf an Solothurn (Gempen 1485/1502, Hochwald 1503). Obwohl Gempen urkundlich erst 1285 erstmals erwähnt wird (Gempenon, 28), scheint es doch weit älter zu sein als Hochwald, wird doch der Ortsname von lat. «campus» abgeleitet $(8,13)$. Die Tatsache, da $\beta$ das anlautende $G$ in Gempen nicht von der althochdeutschen Lautverschiebung (7./8. Jh) erfaßt wurde, läßt außerdem den Schluß zu, daß Gempen bis in diese Zeit noch von einer gallorömischen Bevölkerung bewohnt wurde und erst in der Folge unter alemannischen Einfluß geriet, eine Erscheinung, welche für zahlreiche weitere Siedlungen in Rückzugsgebieten des Juras nachgewiesen wurde (13). Vgl. auch Nuglar, S. 264. Dagegen scheint Hochwald erst in der hochmittelalterlichen Rodungsphase entstanden zu sein (Ersterwähnung 1226: in villa Honvalt, 28), worauf schon die aus einem Flurnamen hervorgegangene Namensform (mundartl. «Hobel») hinweist. Die beiden Hochlandgemarkungen schieben ihre Grenzen bis an die Schicht-

5 Über die Schichtstufen der Ostabdachung vgl. S. $262 \mathrm{ff}$.

6 Herzog $(26,347)$ faßt den Scharten als Zeugenberg auf, möchte jedoch für seine Heraushebung spätere tektonische Bewegungen nicht ausschließen, eine Ansicht, für welche Beweise allerdings noch zu erbringen sind. 


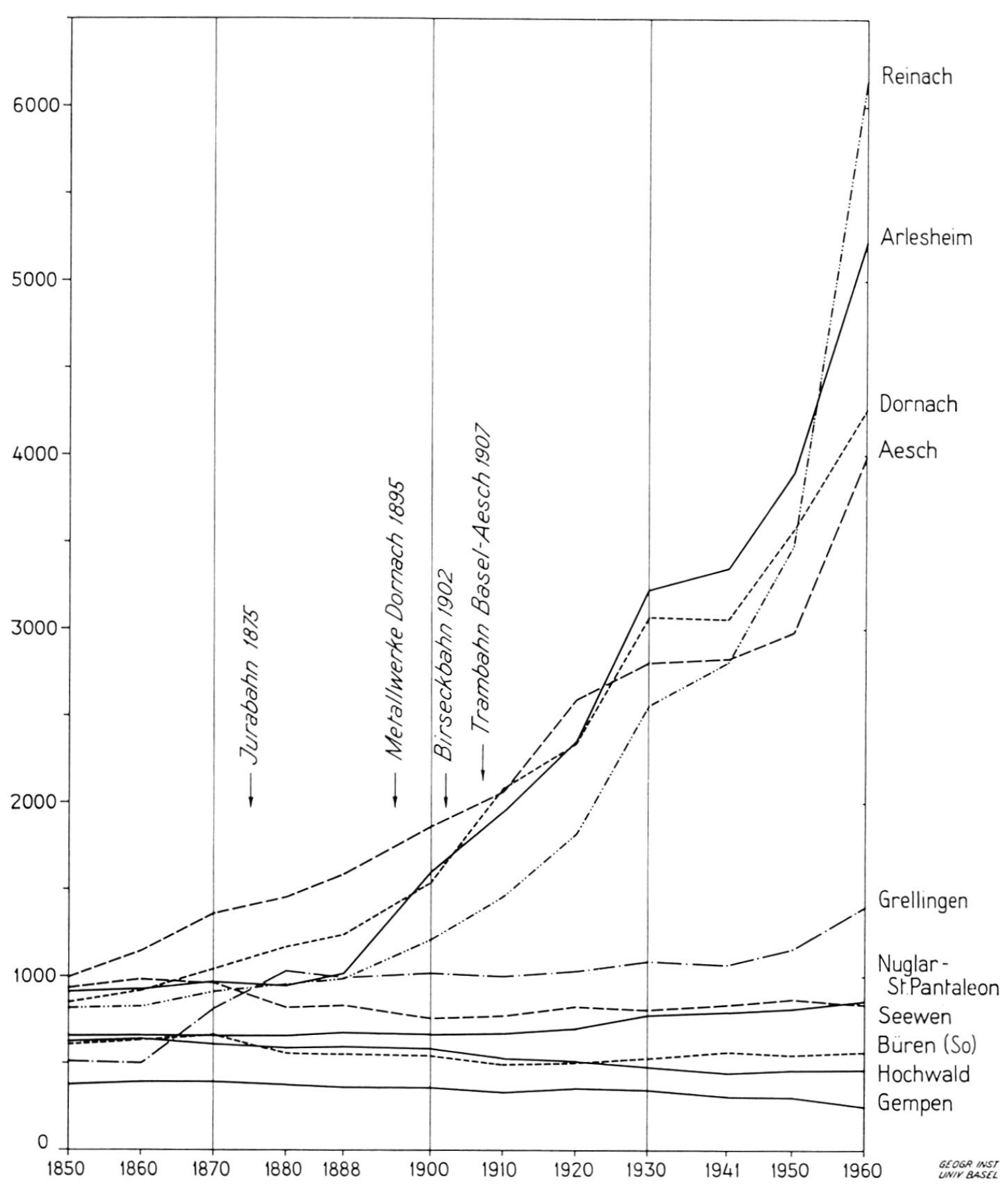

Abb. 8 Bevölkerungsentwicklung 1850-1960. Es lassen sich drei Gruppen unterscheiden: 1. die Gemeinden im Birseck mit stark zunehmenden Einwohnerzahlen, 2. die Gemeinden am Rand des Gempen, wie Grellingen, Nuglar-St. Pantaleon und Büren, mit nur ganz geringer Bevölkerungszunahme und 3. die Gemeinden auf dem Gempenplateau mit abnehmender Einwohnerzahl

stufenkanten vor; lediglich längs der ins Birstal führenden Straßen steigen sie über den Plateaurand in die Oberenden der Täler von Tüfleten und Ramstel hinunter.

Be Infolge ihrer Lage- und Naturungunst nimmt die Bevölkerung der beiden Dörfer seit den 80er Jahren des letzten Jh ständig ab und hat sich in 100 Jahren um mehr als ein Viertel verringert (Abb. 8, 9). Da die ehemals verbreitete Posamenterei seit Jahrzehnten verschwunden ist, die früher bedeutende Lieferung von Brennholz («Hoblemer Wälleli») an die Stadt praktisch aufgehört hat und auch die Herstellung von Hagenbuttengelee («Buttenmost») aus den Hagrosen der Waldränder und Buschhecken (Lesesteinwälle!) zurückgeht, ist die Bevölkerung weitgehend auf die Einkünfte aus der 


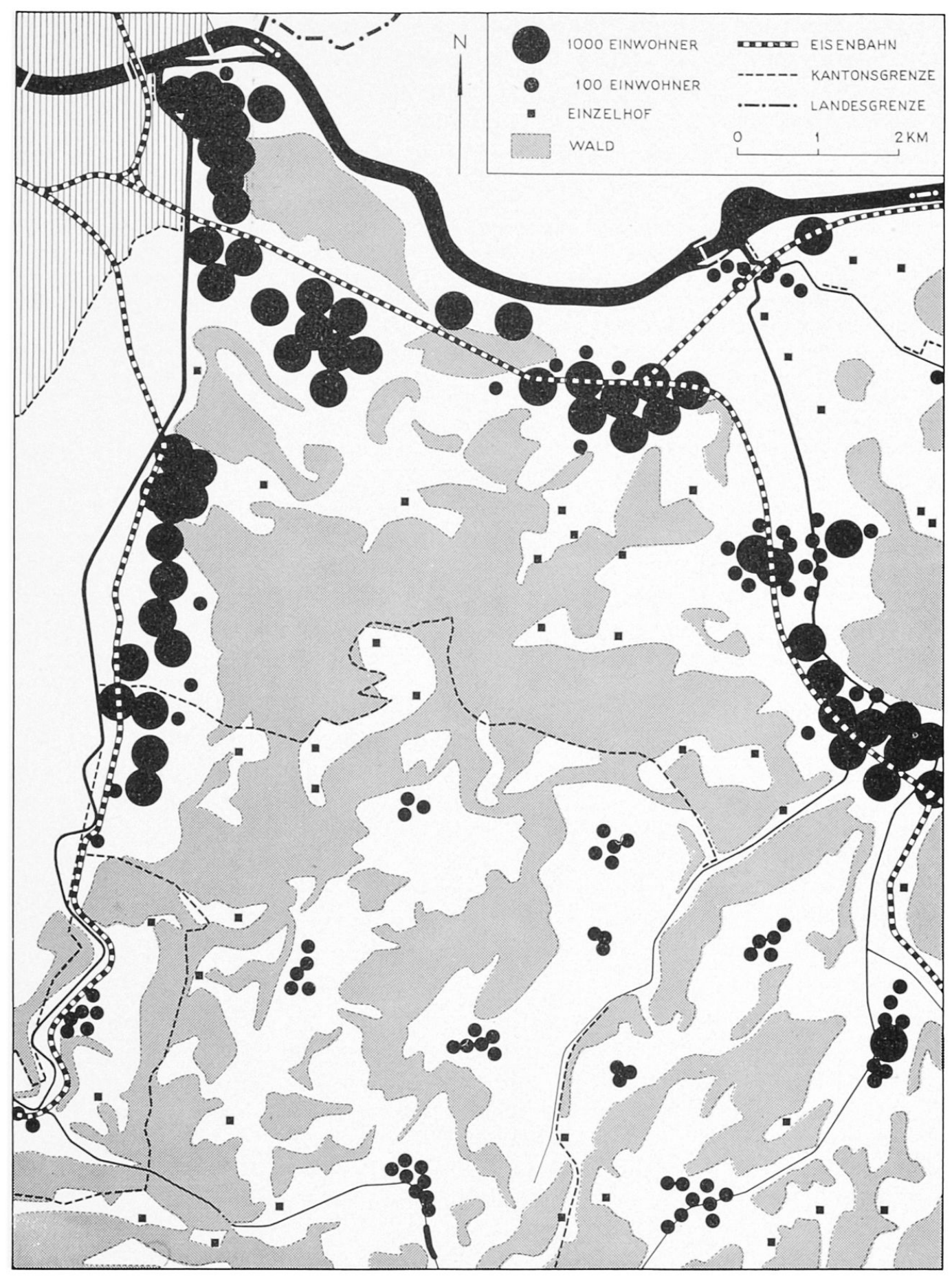

Abb. 9 Bevölkerungsverteilung 1960 (aus H. Annaheim, Basel und seine Nachbarlandschaften, 3. Auflage 1963, Lehrmittelverlag des Kantons Basel-Stadt)

Land- und Waldwirtschaft angewiesen. Jedoch sind die Auspendlerzahlen beträchtlich (Gempen $63=40 \%$ der Erwerbstätigen, Hochwald $55=27 \%, 1950$ ). Als Arbeitsraum steht das Birstal an erster Stelle, mit welchem Gempen durch die kurvenreiche Straße durch den Ramstel und Hochwald durch die über den Dornachberg absteigende Route verbunden ist. Die Karte der Bevölkerungsverteilung dokumentiert den starken Kontrast zwischen dem Regressionsraum des Plateaus und den Ballungsgebieten der umgebenden großen Täler (Abb. 9). 
Der Baubestand der beiden Dörfer ist daher im ganzen stationär und zeigt als Grundtypus das quergeteilte, aus Stein erbaute traufständige Einhaus mit dem typischen Dachknick («Würgi»), einer Form, welche wahrscheinlich vom seit 300 Jahren hier verschwundenen strohgedeckten Ständerhaus übernommen worden ist (50).

Die Wasserbeschaffung bereitete den Höhensiedlungen seit jeher Schwierigkeiten. Gempen $G$ wird durch einige Quellen am Scharten versorgt 7. Hochwald verfügte zufolge seiner Lage inmitten von Kalktafeln ursprünglich lediglich über drei von je einer nahen Quelle gespiesene Laufbrunnen; einige Häuser besaßen außerdem wenig ergiebige, vornehmlich Regenwasser fassende Sodbrunnen. Aussicht auf größere Wassermengen boten allein die an den Plateauflanken austretenden Quellen. So wurde in den 188oer Jahren eine Quelle im obern Tiefental (LK Obertüfleten) in 64o m Höhe mit einem Ertrag von 20 Minutenlitern gefaßt und durch einen Stollen in die Brunnstuben des Dorfes geleitet; diese beliefert jedoch nur die drei bisherigen und einen neuen, vierten Brunnen, so $d a \beta$ in trockenen Perioden das Wasser weiterhin rationiert werden mußte und in Brandfällen gefährliche Situationen eintraten. Erst 1933 konnte eine ausreichende Wasserversorgung mit Hausanschlüssen in Betrieb genommen werden; sie nutzt eine im Banne von Büren sw Mülimatt - am Fuß der Malmschichtstufe — gefaßte Wasserader mit einem Erruß von 300-200o Minutenlitern; von hier wird das Wasser ins Reservoir ö des Hofes Nettenberg, dann dem Dorf und den Höfen Herrenmatt, Ziegelschüren und Nettenberg zugeleitet.

WL Das Bild der Agrarlandschaft wird durch die stark parzellierte Gewannflur der Dreifelderwirtschaft bestimmt, deren Zelgen noch gut zu erkennen sind; bei Gempen liegen sie sw, sö und nö des Dorfes; die Flur von Hochwald gliedert sich in das Außerfeld gegen Gempen, das Oberfeld im NE und das Niederfeld gegen Ziegelschüren. Auffallend ist die intensive Vergrünlandung (über 60\% der agrarischen Nutzfläche, s. stat. Anhang) infolge des Überganges von der Dreifelder- zur Kleegraswirtschaft.

8 Am s Dorfausgang von Hochwald auf dem Sträßchen gegen die Herrenmatt (615 m), einen vor 1766 gegründeten Einzelhof; s weiter durch ein Trockentälchen nach P. 576 und absteigend bis zur Stelle, wo die Talkerbe ö der Hutzmeflue $(617 \mathrm{~m}$, lohnender Blick auf die Grellinger Klus) unvermittelt über der senkrechten Malmkalkwand in die Luft ausstreicht. Am Fuß der Wand entspringt an der Obergrenze des Oxfordien in einer Halbhöhle eine Karstquelle, vermutlich der Austritt der im Bereich der Trockentälchen von Herrenmatt und Chesslet versickernden Niederschläge. Das Wasser der Quelle, welches in trockenen Zeiten im Hangschutt versickert und erst weiter unten wieder erscheint, speist den Dugginger Dorfbach.

R Nun zurück zu P. 576, dann ostwärts durch ein gewundenes Trockental nach P. 602 (Abb.6). Die heute inaktive Vorzeitform ist ca. $50 \mathrm{~m}$ in die Verebnungsflächen von «Uf der Hollen», «Baslerholz» und «Eichenberg» eingesenkt; sie trägt unverkennbar den Stil des fluvial gebildeten Talmäanders mit Prall- und Gleithängen und zwei kurzen Seitentälchen. Von P. 602 aus erblickt man genau gegenüber am Osthang der Mulde von Ziegelschüren den oberen Talabschnitt des Talmäanders im ebenfalls trokken liegenden Wenstel. S ein niedriger Umlaufberg (P. 623). Weiter zum kurz vor 1816 gegründeten Hof Ziegelschüren $(627 \mathrm{~m})$; l. des Weges drei flache Dolinen. Beim Gehöft das einzige oberirdische Rinnsal der Hochfläche, welches nach kurzem Lauf in einer Felsspalte verschwindet.

R Im Bereiche Herrenmatt - Eichenberg lassen sich drei Formkomplexe unterscheiden, welche vermutlich drei aufeinanderfolgenden Abtragsgenerationen entsprechen:

1. die Peneplainflächen Uf der Hollen-Eichenberg in ca. 69o-66om Höhe;

2. die flache, sich über der Falkenflue in $600 \mathrm{~m}$ öffnende Mulde des Falkenfluefeldes (634$599 \mathrm{~m})$;

3. das noch tiefer eingesenkte Trockentalsystem Chesslet-Wenstel, in welches sich auch die Mulde n Ziegelschüren mit dem kleinen Umlaufberg einordnet. Zu diesem Formkomplex gehört vermutlich auch die Wanne von Gempen mit einer Mündungshöhe von $650 \mathrm{~m}$ gegen den Ramstel und die lange Mulde von Hochwald mit ihren östlichen Seitentälchen. Ob der Sattel P. 658 über Obertüfleten (Tiefental) als Abfluß diente, ist ungewiß.

7 Da in den letzten Sommern die Quellen den Bedarf nicht mehr zu decken vermochten, wird gegenwärtig eine Verbindung mit dem Leitungsnetz von Dornach hergestellt, welche in Mangelzeiten in Funktion treten soll. 
Die Komplexe 2 und 3 können sich nur zu einer Zeit gebildet haben, als der Grundwasserspiegel noch im Talbodenniveau lag, also vor Beginn der starken Eintiefung des Birstales. Offensichtlich erfolgte dann die Absenkung der Erosionsbasis und damit des Grundwasserspiegels so rasch, daß die Eintiefung der Tälchen nicht mit ihr Schritt halten konnte. Die Verkarstung setzte mit der ersten Zerschneidung der Peneplain (Phase 2) ein und ergriff mit dem Trockenfallen der Täler des Formkomplexes 3 das ganze Plateau. Sie bildet die Voraussetzung für die ausgezeichnete Erhaltung dieser Vorzeitformen.

Aus der Höhenlage des ältern Deckenschotters im Birstal (ca.37o/8o m) muß, da stärkere quartäre Schiefstellungen nicht nachgewiesen wurden, auf präquartäres Alter der Trockentäler geschlossen werden. Eine Korrelation mit dem hohen Vorkommen des Sundgauschotters s Bettlach $(520 \mathrm{~m}, 52)$ ergibt ein sehr beträchtliches mittleres Gefälle von 2,8\%o. Obwohl die Sundgauschotterplatte anscheinend nach oder schon während ihrer Ablagerung von Bewegungen erfaßt wurde, so deutet dieser hohe Wert doch an, daß die Trockentäler älter als oberpliozän sein dürften. Danach wäre - dies sei im Sinne einer Arbeitshypothese abschließend bemerkt - die Zerschneidung der vermutlich mittelmiozänen Peneplain des Hochlandes im jüngsten Miozän oder ältern Pliozän erfolgt. Nur Untersuchungen in weiterm Rahmen vermögen zu einer Abklärung dieser Fragen zu führen.

9 Von Ziegelschüren auf der Straße entweder nach Seewen oder aber, bei P. 611 von ihr abzweigend, direkt zum Gehöft Bödeli (Gs) im Tale des Seebachs, welches die Südflanke der Gempentafel säumt; die formenreiche Berglandschaft s des Tales gehört

$\mathrm{R}$ dem Kettenjura an, so die überschobene Masse des Buechenbergs s Seewen und weiter der Wisig und der Himmelrieder Homberg. Weiter auf der Straße nach Grellingen bis zum aus mächtigen Malmkalkblöcken aufgetürmten Bergsturzriegel beim Seeloch ( $\mathrm{n}$ Fulnau ).

Die flache Talsohle ö Seewen, «See» genannt, ist der Boden des ehemals 2,5 km langen, durch die Bergsturzmasse gestauten Sees. An seinem obern Ende entstand als Seeufersiedlung das Dorf Seewen $(544 \mathrm{~m})$, dessen alter Dorfkern nö des von der Kirche gekrönten Spornes liegt. Beim Einfluß des Seebaches in den See lagen ursprünglich lediglich die Mühle und einige Häuser. Nach der Trockenlegung des Sees dehnte sich die Siedlung etwas gegen den Talboden hin aus. S des Dorfes der im Jahre 1870 vom

S Basler Wasserwerk angelegte, bei starken Gewitterregen als Ausgleichsbecken für den Seebach dienende «Baslerweiher».

$G \quad$ Nach ersten Versuchen zur Absenkung des Sees (1+88) wurde gegen Ende des 16. Jh ein $200 \mathrm{~m}$ langer Stollen, das Seeloch, durch die Bergsturzmasse gegraben. Wenn dadurch der See auch weitgehend entleert werden konnte, so blieb doch der alte Seeboden sumpfig und ergab lediglich Riednutzung, ein Zustand, den auch die Erweiterung des Seelochs im Jahre 1753 nicht zu ändern vermochte. Erst die im Jahre 1919 vom Landbesitzer, dem Baselstädtischen Wasserwerk, durchgeführte Korrektion des Seebaches und die Drainage der Talebene schufen die Voraussetzungen zu einem ertragreichen Acker- und Gemüsebau.

R Nun südwärts bergan zum Hof Fulnau. Über dem Hof ist die Abrißnische des Bergsturzes und namentlich deren Westflanke gut zu erkennen.

Die Entstehung des Bergsturzes an dieser Stelle ist eine Folge der spezifischen morphologischen Situation: Die Nahtstelle zwischen Tafel- und Faltenjura findet sich in einer schwach ausgeprägten Synklinale am Nordfuß der Homberg-Wisig-Antiklinale in der Linie Stollen — Eigen. In die leicht nördlich ansteigenden Gesteinshorizonte des anschließenden Tafeljuras ist das untere Seebachtal eingeschnitten, wobei es sich ostwärts immer mehr der Wisig-Kette nähert. An der Stelle, wo der Seebach den Fuß der Antiklinale erreicht und bis in die Oxfordmergel angeschnitten hat, ist der Bergsturz erfolgt, wobei die Korallenkalkplatte auf den Mergeln und Tonen des untern Malms zu Tal gefahren ist.

Nun zurück zur Straße und direkt nach Grellingen oder aber über den Hof Eigen mit Einblick in die Halbklus von Bärtel, welche beidseits von den Korallenkalken des Nordschenkels der Wisig-Kette eingefaßt wird, weiter über Stollen und Abstieg zur von Himmelried nach Grellingen führenden Straße.

Im Seetel ( = Seetal, auch Pelzmühletal, nach einer ehemaligen Mühle) befinden sich die wichtigsten unter den beidseits das Tal überhöhenden Malmkalkflühen austretenden Quellen des jurassischen Teiles der Basler Wasserversorgung («Grellinger Werk»); dazu gehören auch die ebenfalls vornehmlich an das Oxfordien gebundenen Quellen des nahen Kaltbrunnentales und von Oberäsch-Angenstein. Die Quellen leiden 
unter großen Erguß-Schwankungen (zwischen 2500 und $12000 \mathrm{~m}^{3} / \mathrm{Tag}$ ) und trüben sich zudem nach starken Niederschlägen. Die seit dem Jahr 1865 für Basel gefaßten Grellinger Wasser werden deshalb in dem 1904 auf dem Bruderholz bei Basel erstellten Reservoir filtriert. Gegenwärtig werden kaum noch 10\% des Wasserbedarfs von Basel aus diesen Quellen bezogen; der Rest wird aus den Grundwasserströmen von Wiese (Erlenpumpwerk) und Rhein (Hard bei Muttenz) gewonnen.

\section{Varianten (Teilstrecken) zu Hauptroute 1 (Abb. 1)}

Teilstrecke Ia

Muttenz - Wartenberg - Egglisgraben - Stollen

1 Der Dorfkern von Muttenz (291 m) liegt im Winkel zwischen Wartenberg und Rütihard an der Mündung eines kleinen, in die N-Abdachung der Gempentafel eingesenkten Talsystems auf dem flachen Schwemmkegel, welchen das Talgewässer auf das oberste Niveau der Niederterrasse ausgebreitet hat. Die Lage ist im Hinblick auf die Wasserversorgung, die klimatische Begünstigung und die rasche Erreichbarkeit der verschieden ausgestatteten Räume des Gemeindebannes vortrefflich.

Der Ortsname (1027 Mittenza, vermutlich von lat. «mutatio»: Pferdewechselstation) und zahlreiche Funde weisen auf keltorömische Entstehung der Siedlung hin, welche damals vermutlich in der Nähe der Überlandstraße lag. Diese berührt den alten Dorfkern nicht, sondern zieht vom Birsübergang bei St. Jakob im $\mathrm{N}$ des Wartenbergs vorbei.

Im Mittelpunkt des fünfstrahligen Dorfkernes liegt die ehemals vom Dorfbach umflossene Burgkirche mit einem bis $7 \mathrm{~m}$ hohen Mauerring, zwei Tortürmen und dem massigen, 163o erhöhten Kirchturm (38). Der Kirchenpatron, der Hl. Arbogast, erinnert an die engen frühmittelalterlichen Beziehungen der Region mit Straßburg 8; die Straßburger Bischöfe waren bereits vor dem 8. Jh im Besitze von Muttenz und des Wartenbergs, der später an die Froburger und Homburger, im 14. Jh an die ritterliche Familie der Münch von Münchenstein und schließlich 1515 an Basel überging. Im Innern der Kirche und des an die Wehrmauer angelehnten Beinhauses sehenswerte spätmittelalterliche Wandmalereien; im Kirchhof eine Kalksteinsäule, welche der Vorhalle einer ehemaligen Wegkapelle beim Schänzli (über der Birs) entstammt und vielleicht der Rest eines römischen Meilensteins ist. Neben dem Beinhaus die einzigartige Sammlung von Grenz- und Gütersteinen aus dem Muttenzer Bann, welche nach der Güterregulierung (190331) angelegt wurde und die vielfältigen ehemaligen Besitzverhältnisse an der zersplitterten Gemeindeflur dokumentiert (16-19).

Fünf Siedlungszeilen führen von der Wehrkirche zu den einzelnen Wirtschaftsflächen der Gemeinde (Abb. 10) :

1 Die Hauptstraße, eine doppelte Bachzeile (Bach heute eingedeckt), hinaus zu den Feldern und ehemaligen Reben (Feldreben) des Niederterrassenfeldes und zum Waldgebiet der Hard, heute zur Tramstation und zum Bahnhof;

2 die Gempengasse längs des Dorfbaches zu den Grundstücken im Talraum südlich des Wartenbergs und hinauf zu den Wäldern am Abhang des Plateaus;

3 die Burggasse zu den Rebgütern am Wartenberg;

4 die Geispelgasse (Geispel = Gänsebühl, s des Dorfes, Rest älteren Deckenschotters, 370-385 m) zu den fruchtbaren, auf Lößlehm stehenden Ackerfluren des Rütihardplateaus ('Terrasse des jüngern Deckenschotters, 340-350 m);

5 die Baselgasse (alte Straße nach Basel) wieder auf die Niederterrassenflur.

Nach dem Bahnbau (1854 Basel-Liestal, 1858 nach Olten) und dem Trambahnanschluß an Basel (1921) setzte sich ein immer stärkerer Wandel der Agrargemeinde zur Industrie- und vorörtlichen Pendler-Wohngemeinde durch (1960: $3 \%$ in der Landwirtschaft Tätige, 182o Pendler nach Basel = 35\% der Erwerbstätigen). Die Bevölkerung nahm von 167o (1850) auf 11971 (1960) zu, in welchen Zahlen die Einwohner des bis zum Jahre 1875 zum Muttenzer Banne gehörenden Birsfelden nicht berücksichtigt sind; seit 1815 hat sich dieser Ort von einer nur 5 Häuser zählenden Kleinsiedlung zu einem Vorort mit über 1o ooo Einwohnern entwickelt (vgl. Abb. 9).

8 Arbogast, seit ca. 55o Bischof von Straßburg, Hauptbegründer des Christentums im Elsaß, Erbauer des ersten Straßburger Doms. 


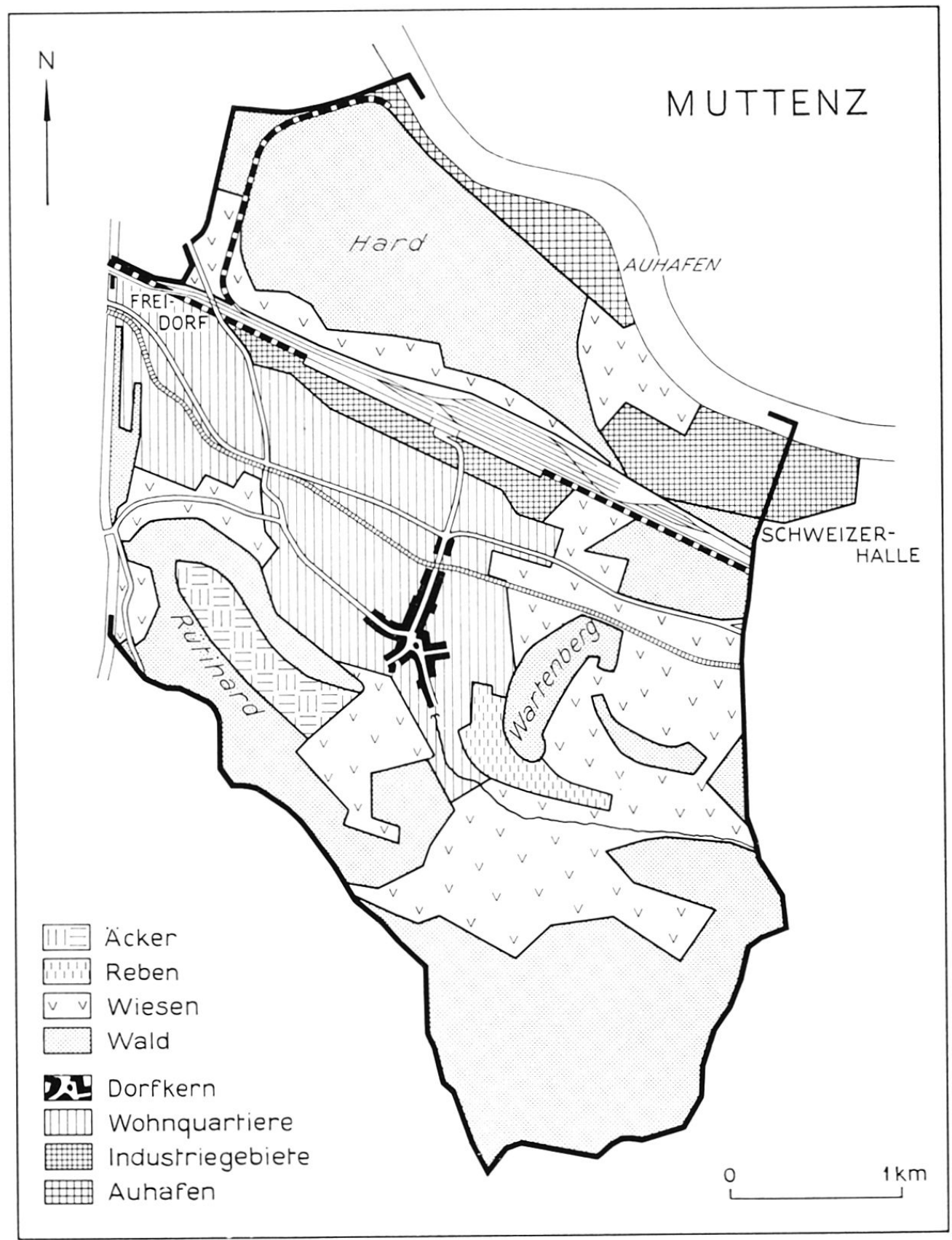

Abb. 1o Nutzungsplan der Gemeinde Muttenz (aus H. Annaheim, Basel und seine Nachbarlandschaften, 3. Auflage 1963, Lehrmittelverlag des Kantons Basel-Stadt)

Die mannigfaltige Industrie konzentriert sich längs der Bahnlinie, an der Birs gegenüber St. Jakob und am Rhein im Anschluß an die Saline Schweizerhalle (Gewinnung von Salz aus der Anhydritgruppe seit 1836). Auf Muttenzer Boden liegen außerdem der große Verschiebebahnhof Basels und ein Teil der Rheinhafenanlagen (Auhafen). Über das Niederterrassenfeld zwischen Rütihard und Bahnlinie und das ehemalige Rebgelände am Wartenberg breitet sich ein immer dichter werdendes Netz von Wohnquartieren aus. Der historische Dorfkern mit der Wehrkirche und den vorwiegend im 17. und 18. Jh erbauten Bauernhäusern vom Typ des quergeteilten Einhauses, welches hier meistens mit Weinkellern (Rebbau am Wartenberg!) versehen ist, soll nach dem neuen Bebauungsplan in seiner Form erhalten bleiben, wenn auch seine ursprünglich agrarische Funktion weitgehend verlorengegangen ist (39). 

Ruine passierend, zum Rundturm (Aussicht!) am Südende des Rückens (479 m). Der Wartenberg trägt seinen Namen zu Recht.

Vielleicht schon in keltischer Zeit ein Refugium, erhob sich auf dem nördlichen Felskopf eine römische Warte zum Schutze der Straße Augusta Raurica-Basilea, später eine fränkische Befestigung, auf deren Grundmauern die spätere Burgenanlage errichtet wurde. Die Straßburger Bischöfe sicherten ihr Gebiet durch den Bau einer Burgenreihe, indem sie die Vordere Burg durch eine Mittlere und Hintere (im S) ergänzten. Aus der Hand der Münch von Münchenstein, welche seit Ende des 1t. Jh Muttenz besaßen, kam das Dorf mit den Burgen 1515 an Basel; die Burgen waren damals offenbar schon nicht mehr bewohnbar, zerfielen in der Folge, wurden jedoch seit 1952 teilweise restauriert $(17 ; 37)$.

Der Kamm - strukturell eine abgesunkene Scholle - ist eine durch ö einfallenden

R Hauptrogenstein gekrönte Härterippe, deren W-Flanke steil zu den sanfter geböschten Hängen im untern Dogger, Lias und Keuper abfällt (26). Die E-Flanke wird eindrücklich durch den tektonisch bedingten Gesteinswechsel geprägt: Der Wald steigt hier bis zu einer NNE-streichenden Bruchlinie ab; die Acker- und Wiesenfläche ö davon liegt in den Mergeln des obern Doggers einer weitern, schmalen Scholle; jenseits einer zur ersten parallel verlaufenden Bruchlinie (bei Hinter Wartenberg) ein gerodeter Höhenrücken, der seine weiche, am N-Hang von Rutschungswülsten gekennzeichnete Formung Keupermergeln verdankt, welche von einer dünnen Decke von Liaskalk und -mergel bedeckt sind. Das zum Hof Egglisgraben aufsteigende Tal ist eine Ausräumung im Keuper, welche im WE-streichenden, schon vor der Rheintalflexur angelegten Adlerhofgewölbe eingetieft ist. Im S steigen die von einem Schuttmantel überzogenen, ebenfalls zahlreiche Rutschungen zeigenden Wiesen- und Waldhänge (Steinbrüche im Hauptrogenstein) zur strukturell zerbrochenen Doggerplatte der nördlichen Gempenhochfläche an. Jenseits des Hochrheintals mit seinen Niederterrassenfeldern der Dinkelberg (Dinkel = Spelz, eine Weizenart), eine aus Muschelkalk mit eingeschalteten Keupergräben aufgebaute Plateaulandschaft agrarischen Charakters; dahinter der Hochschwarzwald.

3 Vom Wartenberg-Rundturm ö absteigend und auf dem anschließenden Höhenrücken zum im Jahre 1813 entstandenen Ausbauhof Egglisgraben (Gs), dann entweder $\mathrm{s}$ ansteigend zum Horn und weiter zur Schauenburgflue $(663 \mathrm{~m})$, einer Malmkalkschichtstufe, auf der im vergangenen Jahr ein gallo-römischer Tempel von Th. Strübin (Liestal) ausgegraben worden ist. Über die Peneplain (s. S. 251) nach Stollen (638 m; Anschluß an die Hauptroute I) oder aber ostwärts um den Plateausporn des Horns herum nach Neu-Schauenburg (Anschluß an Teil route Ib, S. 261).

\section{Teilstrecke Ib}

\section{Pratteln - Schauenburgflue - Stollen}

S 1 Pratteln $(296 \mathrm{~m})$, wie Muttenz vermutlich schon keltorömische Siedlung (1101 Bratello, vermutlich keltorömische Namensform; 28). Auch nach Lage und Siedlungscharakter stimmt Pratteln mit dieser Siedlung weitgehend überein.

Der alte Dorfkern entwickelte sich ebenfalls an der Mündung eines Tälchens auf dem Schwemmkegel des Talbachs; im Siedlungszentrum die ehemalige, heute ihrer Mauern beraubte Burgkirche, deren Schutzpatron St. Leodegar auf den Einfluß des Vogesenklosters Murbach hinweist, welches sich hier schon früh an der Route nach Luzern 8 festgesetzt hatte. Bei der Kirche kreuzen sich die beiden Siedlungsachsen, von denen die eine dem Talbach entlang ins Tälchen und auf die Terrassenfläche hinaus vorstößt, die andere dem Hangfuß entlang die Überlandstraße begleitet, welche heute die Siedlung in weit ausholendem Bogen umfährt. Im Gegensatz zu Muttenz besitzt Pratteln eine ehemalige Burganlage, ein am N-Rand des Dorfkerns errichtetes Weiherhaus, welches nach dem Erdbeben von 1356 anstelle des zerstörten Bergschlosses Mad-

$P$ len (auf der höchsten Stelle des Adlers s des Dorfes; «Adler» eine volksethymologische Umdeutung aus «Im Madlen») erbaut wurde; seit 1773 in Besitz der Gemeinde, welche den Wassergra-

8 Seit 8 to besaß hier Murbach das Benediktinerkloster St. Leodegar «im Hof» (an der Stelle der heutigen Hofkirche). 
ben auffüllen und die Ringmauer niederreißen ließ. Pratteln kam 1521, Muttenz 1515 an Basel, zwei Erwerbungen, welche für die Stadt von größter Bedeutung waren, stellte sie doch damit die räumliche Verbindung mit ihrem ausgedehnten Besitz im Ergolzgebiet her.

WJ In vermehrterem Maße noch als Muttenz hat sich Pratteln seit dem Eisenbahnzeitalter (1854 Basel-Liestal, 1858 nach Olten, 1875 Linie nach Zürich) dank seiner günstigen Lage im Knotenpunkt der Linien nach Olten, Zürich und Schaffhausen zur Industriesiedlung entwickelt; längs der Bahn haben sich zahlreiche industrielle Großbetriebe angesetzt (Eisenkonstruktionen Buss, Pneu-, Persil-, Waggonfabrik, Handelsbetrieb des VSK usw.) und auf Pratteler Boden liegen die Saline Schweizerhalle (Salzgewinnung seit 1836) und salzverarbeitende Industrien, welche über einen Bahnanschluß mit der Station Pratteln verfügen. Infolge der geringeren Breite der oberen Niederterrasse ist der Waldgürtel der Hard hier schon frühzeitig gerodet worden und die moderne Überbauung mit Industrie- und Wohnquartieren dichter als in Muttenz. Bedeutende Pendelwanderung nach Basel, mit welchem Pratteln seit 1922 durch eine Trambahn verbunden ist.

2 Zunächst dem Talbach folgend hinauf zum 1784 gegründeten Hof Ebnet, einem der noch vor Aufhebung des Flurzwanges (1829) gegen den Protest der Gemeinde von Basler Bürgern errichteten Landwirtschaftsbetriebe (Höchenrain 1695, Maienfels 1726 , Schönenberg 1767, Lachmatt 1795, Egglisgraben 1813). Der leicht ansteigende, Reste von Rebäckern tragende Hang zeigt die weichen Formen der Liasmergel und -kalke, welche leicht ostwärts fallen; im E des Talbachs zunächst Opalinustone, dann schuttüberdecktes Bajocien und, den Adlerkamm aufbauend, Hauptrogenstein. Über Keuperund Schutthänge hinauf nach dem Landgut Neu-Schauenburg $(480 \mathrm{~m})$ und über einen Hauptrogensporn zur Ruine Schauenburg (601 m).

Die schon um 1500 zerfallene Burg steht auf einem von der Schauenburgflue abgesackten Klotz von Korallenkalk. Auch am Platze von Neu-Schaucnburg stand eine diesen Namen tragende 3urg, welche jedoch durch das große Erdbeben zerstört wurde; später wurde an diesem Platz ein Bruderhaus gebaut, das dann in ein bis 1650 bestehendes Beghinenhaus umgewandelt wurde; das in der 1. Hälfte des 18. Jh eingerichtete Bad hatte keinen langen Bestand, und das Gut wurde zum Landwirtschaftsbetrieb. $\mathrm{S}$ in der Tiefe das Schauenburgerbad, ein ehemaliges Solbad, heute Gasthof.

R Nun über großblockige Sturz- und Sackungsmassen hinauf zur Schauenburgflue $(658 \mathrm{~m})$, deren Korallenkalk-Schichtstufe zwischen P. 658 und dem Chleiflühli bis zum w einer gedoppelten Bruchlinie folgenden Hauptrogenstein ausgebrochen ist. Über die Hochfläche bei Gmeinacher - die Peneplain streicht hier glatt über drei Schollen hinweg (s. Karte in 26) - nach Stollen (Anschluß an Hauptexkursion I).

\section{Teilstrecke Ic:}

\section{Falkenflue - Duggingen}

R Nach dem Besuch des eingesenkten Trockentals (Chesslet, Hauptroute I, 8, S. 256) zurück zu P.576 s Herrenmatt, s auf die Korallenkalkschichtstufe der Falkenflue $(624 \mathrm{~m})$ mit schönem Blick in die Birsklus und auf das E-Ende der asymmetrisch gebauten Blauenkette mit schräg zur Eggfluh aufsteigendem S-Schenkel (Malmkalk) und nach einer Abtragslücke in der Schichtrippe der Ruine Pfeffingen steil einfallendem, sich dann nordwärts abflachendem N-Schenkel; n Grellingen der Faltenkern (Dogger).

s Abstieg über den Hof Luegi (Luegmatt, $442 \mathrm{~m}$ ) nach Duggingen (331 m); das auf der schmalen Niederterrasse liegende Dorf zeigt einen 'T-förmigen Grundriß: doppelte Bachzeile längs des Dorfbaches, Wegzeile längs der alten Straße von Aesch nach Grellingen, dem Terrassenrand folgend. 
34 Hauptroute II (mit Benützung eines PW, Abb. 1)

1 Über Arlesheim (S. 247 ff.; 32.3 u. 4) und Dornachbrugg S. $2+2$ ff.; 32.1 ) nach Dornach («Oberdornach», $338 \mathrm{~m}$ ), auf der Hochterrasse, welche mit Löß und mit Alluvionen des Baches aus dem Ramstel bedeckt ist.

Funde aus der La Tène-Zeit und die Ortsnamenform (《-ach»-Suffix, lat. «fundus turrania:us», Gut des Turranius) scheinen auf keltische Entstehung hinzudeuten; Ersterwähnung 1223 Tornacho. Die Siedlung kam 1502 mit dem Schloß Dorneck an Solothurn. Die an Obstbäumen außerordentlich reiche, ursprünglich weite Rebareale umfassende Flur ist stark durchsetzt mit Villen und Einfamilienhauszeilen namentlich gegen den Goetheanum-Hügel (S.249), so daß das bäuerliche Element auch im Dorfteil immer mehr zurücktritt; zahlreiche Bewohner finden in den Metallwerken Beschäftigung (S.246). Die alte Dorfkirche dient als Heimatmuseum der Ämter Dorneck und Thierstein (sehenswerte Sammlungen).

Fahrt zur Ruine Dorneck (497 m, Gs) auf einer durch ein Oxfordkombentälchen isolierten Korallenkalkrippe ${ }^{9}$; 1360 erstmals erwähnt, nach ihrer Erwerbung von Solothurn infolge ihrer strategischen Bedeutung zur starken Festung ausgebaut, 1798 zertört; $85 \mathrm{~m}$ tiefer Sodbrunnen (30).

Rundblick vom Turm (vgl. S.249; 32.4); im SE die der Gobenmatt (S.250) ähnliche Ausräumung des Ramstel, welche rechtsseitig von zwei bis auf den Dogger entblößten Schollen flankiert wird, über denen sich der Block der Schartenflue - die tektonische Fortsetzung der Burgenrippe von Dorneck - heraushebt; im S der Ausräumung führt der geschlossene Malmkalkschenkel des Dornachbergs in bewaldetem Aufschwung bis zur Plateauhöhe.

2 Von der Ruine Dorneck zurück nach Dornach und durch den Ramstel aufwärts; s der durch Steinbrüche angeschlitzten Doggerschollen erscheint der sich westwärts absenkende, in einem Felskopf endende Ingelstein, dessen massige Skulptur durch Korallenkalk geprägt ist. Vom n benachbarten Waldrücken (oberer Dogger, Schartenscholle) ist die Ingelstein-Tiefscholle durch die nach Gempen hinaufziehende Verwerfung getrennt. Von der Spitzkehre P. 583 Aufstieg zur Glitzersteinhöhle, einer der Karsthöhlen des Plateaus; Eingang in ca. $640 \mathrm{~m}$ am Fuße der Fluh; der bogenförmige Höhlengang (Abb. 11) durchsetzt den Korallenkalk ca. $50 \mathrm{~m}$ über dem wasserstauenden Horizont des untern Malms (Oxford) und ist heute im Stadium der Versinterung; er muß während einer frühern Phase der Eintiefung des Ramstels entstanden sein (25).

3 Von Gempen in 15 Min. zu Fuß auf die Schartenflue (S. 251; 32.6). Dann zurück nach Gempen und Fahrt nach Hochwald (S. 253; 32.7). Von hier nach Hauptroute 1 (S. 256; 32.8) zu Fuß über Herrenmatt zum Ende des Trockentälchens sw P. 576, dann durch den trockenen Talmäander nach P. 602 und auf einem Feldweg zurück nach Hochwald (ca. 1-11/2 Stunden). Weiterfahrt Richtung Seewen; beim Erreichen des Talbodens scharf nach r. und auf der Straße gegen Grellingen bis zum Bergsturzriegel beim Seeloch; zu Fuß hinauf nach Fulnau (Blick in die Abrißnische des Bergsturzes) und zurück (20 Min.) (S. 257 ; 32.9). Nun nach Seewen (S. 257).

4 Von Seewen weiter auf der Straße nach Büren; zunächst über die wasserscheidende R Höhe $(597 \mathrm{~m})$ zwischen Seebach- und Oristal, dann auf der Ostabdachung der Gempentafel, einer modellartig entwickelten Schichtstufenlandschaft, nach Büren. Büren (441 m) liegt am Ausgang eines mächtigen, in den E-Abfall des Plateaus vorgetriebenen, quellenreichen Ausräumungskessels, der bis in die Höhe der Landterrasse des obern Doggers hinunterreicht. Er wird von den bewaldeten Steilhalden und Fluhreihen der Malmschichtstufe (Rauracien, Sequan) eingefaßt, welche nach unten in die weniger geböschten, gerodeten Halden in den Oxfordmergeln übergehen; diese sind weithin von den an den Malmkalken ansetzenden Schuttschleppen bedeckt. Die Oxfordquellen,

9 Am Südende der Rippe (614 o75/258 425) Reste des unterstampischen Kliffes, das auf $100 \mathrm{~m}$ durchbrochen wurde; in der Durchbruchstelle ein aus Korallenkalk bestehendes Meeressand-Konglomerat ( 27 ). 
GLITZERSTEINHOHLE

aufgenommen von John Haller

1948

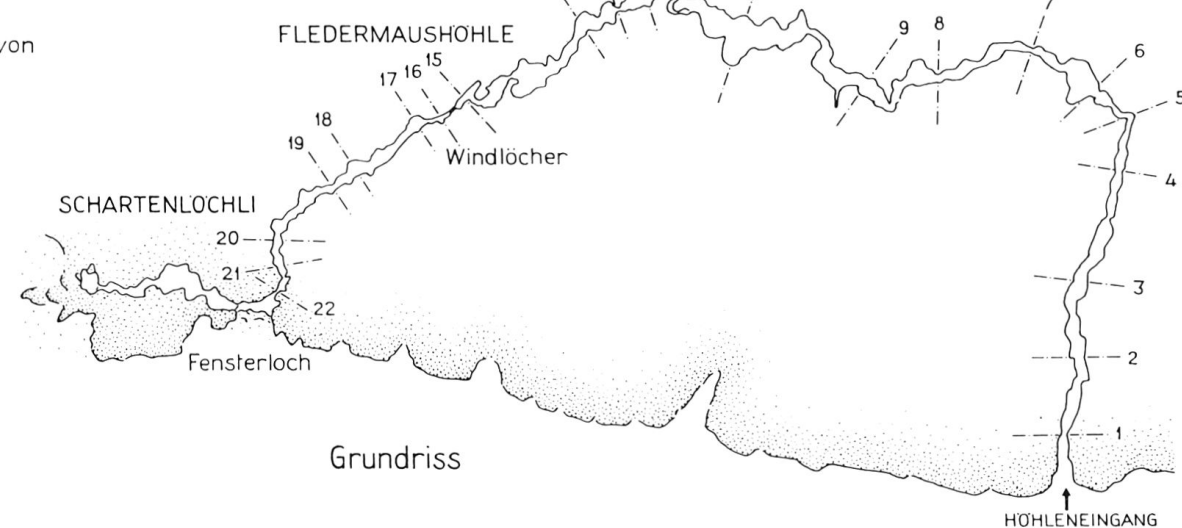

Querschnitte und Längenprofil

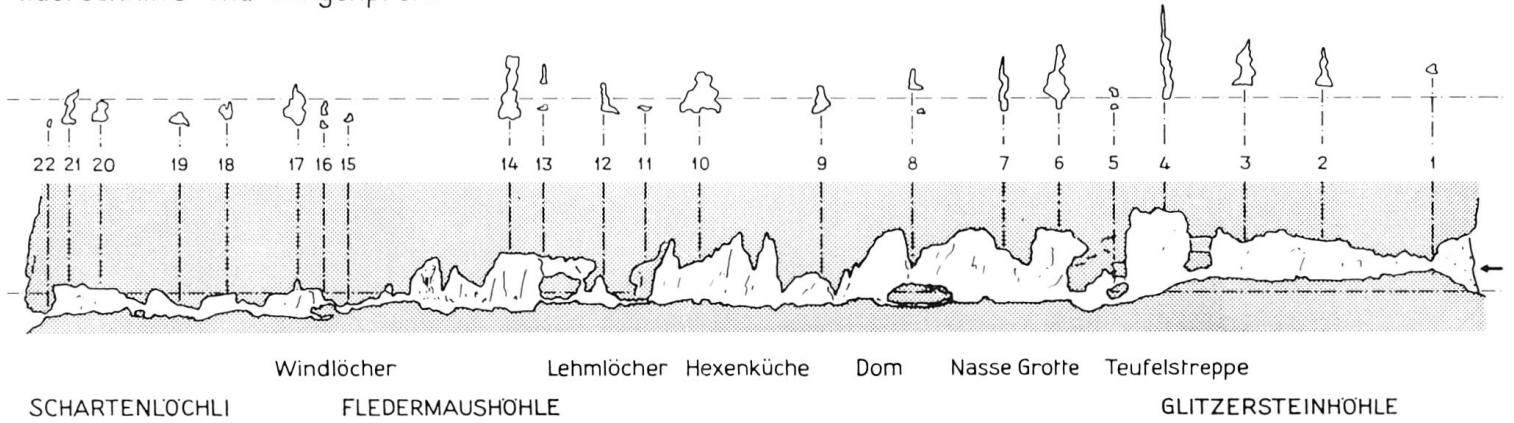


deren Wasser früher auch zur Wiesenbewässerung verwendet wurde, versorgen Büren mit Nutzwasser, so der Duftbach (Duft $=$ Kalktuff) $w$ des Dorfes in $570 \mathrm{~m}$ Höhe (Schüttung 60-500 Minutenliter); eine der Quellen beliefert Hochwald mit Wasser (S. 256). Die beidseits des Kesselausgangs vorspringenden Sporne (S: Chöpfli, Horn, $\mathrm{N}$ : Schlimmberg, auf T. A. Schlingenberg) verdanken ihre Akzentuierung einer SSWNNE streichenden, zweigeteilten, gegenüber dem w anschließenden Komplex abgesunkenen Scholle; deren widerständiger Korallenkalk wird an der westlichen der beiden Bruchlinien vom Oxfordmergel abgelöst, womit die Ausweitung des Kessels einsetzt.

$S$

Der Kern des Dorfes (1194 Buoron, von ahd. «bur», Wohnstätte, 28) ist eine WE gerichtete doppelte Bachzeile, an welche sich längs der Flurwege weitere Hauszeilen angefügt haben. Talwärts fällt eine inmitten eines heute trockenen Weihers stehende, rechtwinklig zusammengebaute Häusergruppe auf; sie steht auf den Mauern eines ehemaligen Wasserschlosses, welches wahrscheinlich nach dem großen Erdbeben von 1356 zum Ersatz der Burg Sternenberg (Reste auf dem Sporn n des Dorfes) erbaut wurde, aber nur bis 1600 als festes Haus gedient hat. Vgl. den FN Schloßmatt ESE des Dorfes! Die Einteilung der Feldflur in die drei Zelgen ergab sich aus der klaren Raumgliederung des Gemeindebannes: Oberfeld im Kessel gegen Gempen, Außerfeld im SE, Hinterfeld gegen St. Pantaleon. Die Vergrünlandung hat hier schon um die Mitte des 19. Jh zusammen mit der starken Ausbreitung des Kirschobstbaues eingesetzt; der in Südexposition erstaunlich hoch hinaufsteigende Rebbau (Sternenbergreben bis gegen $550 \mathrm{~m}$ ) ist stark zurückgegangen. Güggel- und Kohlerhof sind anscheinend erst in der

S zweiten Hälfte des letzten Jh ausgebaut worden, fehlen sie doch auf ältern Karten.

R Weiter auf schmalem Feldsträßchen über die aus oberm Dogger (Callovien) und Hauptrogenstein aufgebaute Landterrasse nach St.Pantaleon (489 m) und Nuglar $(487 \mathrm{~m})$. Sie wird von der ein- und ausbiegenden Malmkalkschichtstufe überhöht, deren Sockel aus leicht ausräumbaren Oxfordmergeln besteht; auf ihnen sind da und dort ganze Malmpakete abgerutscht, wie an der Schauenburgflue oder bei Nuglar, wo der Stufenfuß von einem größern Bergrutsch überdeckt wird, dessen unruhige Topographie bis ins Dorf hineinreicht. Die n Nuglar gelegene, ebenfalls aus Hauptrogenstein bestehende Landterrasse des Munifeldes (LK: Muni) liegt ca. $100 \mathrm{~m}$ höher als diejenige von Nuglar, weil sie einer etwas höher gestaffelten Scholle angehört; die trennende Bruchlinie begleitet den südlichen Hangfuß des Muni-Plateaus. Die Landterrasse der beiden Dörfer geht nach unten in die steilen Hauptrogensteinhänge über, welche in den schmalen Kanal des Oristales abfallen. Von diesem aus haben die Gewässer schmalsohlige Talgräben in die Landterrasse eingekerbt und sie in p einzelne eng abgezirkelte Flur- und Siedlungsbereiche zerlegt, auf welchen sich die ländlichen Siedlungen Nuglar und St. Pantaleon entwickelt haben; sie sind in einen einzigen Gemeindebann eingefügt, der sich wie jener von Büren trefflich an die naturräumliche Ordnung anschmiegt. Beide Gemarkungen finden ihre Grenzen einerseits in der Höhe des Traufs der Malmschichtstufe, andererseits in der Tiefe des Oristales; Büren greift lediglich da, wo seine durch die Kehle über Luterbrunnen aufsteigende Verbindung mit Hochwald den Trauf erreicht, noch etwas auf das Hochland hinauf.

S Die Tatsache, daß die Gemarkungen von Gempen, Hochwald, Büren und NuglarSt. Pantaleon gleiche Größenordnung aufweisen und - mit Ausnahme der letztern jede von ihnen eine einzige, zentral gelegene Dorfsiedlung besitzt, läßt vermuten, daß auch der Bann von Nuglar-St. Pantaleon von einer Siedlung aus geschaffen wurde.

Funde römischer Münzen und der ON (Ersterwähnung 1146 Nugerol, von lat. «nucarolium», Nußwäldchen; 13) dürften die Entstehung Nuglars in keltorömischer Zeit belegen; es ist wohl kein Zufall, daß diese älteste Siedlung im Gemarkungszentrum und zugleich auf dem ausgedehntesten der drei Landterrassensektoren liegt, über welche sich der Gemeindebann ausdehnt. Sie verfügt außerdem über eine voll ausgebaute Dreizelgenflur (Hinterfeld im N, Unterfeld im E und Außerfeld im S des Ortes; 21). Im Gegensatz dazu findet man bei St. Pantaleon lediglich 
ein oberes und ein unteres Feld; die Siedlung hat sich vermutlich aus jenem frühalemannischen Ort entwickelt, von welchem der Name «Liela» überliefert ist. Der heutige ON geht auf die im hohen Mittelalter weitherum berühmte Wallfahrtskirche St. Pantaleon zurück, welche in der Folge Pfarrkirche nicht nur für Nuglar, dessen Dorfkirche zur Kapelle reduziert wurde, sondern bis zur Reformation auch von Lupsingen und Seltisberg wurde. Während sich das immer volkreichere Bauerndorf Nuglar in eine Geländemulde des vom Hollen abgebrochenen Schuttstromes einschmiegt, gruppieren sich die wenigen Häuser von St. Pantaleon um die auf weithin sichtbarer Höhe liegende Kirche.

Der FN Winzligen (Winslingen), welcher mit einer Flur am obern Ende des Talgrabens s Nuglar verknüpft ist, weist auf einen weitern, vermutlich während der Landnahmezeit bezogenen Siedlungsplatz hin, welcher in der Folge jedoch wieder aufgegeben wurde; Anla $\beta$ zur Wahl des Platzes gab wahrscheinlich die heute gefaßte Quelle, welche direkt über der Flur Winzligen in $580 \mathrm{~m}$ Höhe entspringt (LK: Brunnstube in $570 \mathrm{~m}$ ).

Von Nuglar hinab ins Oristal und nach Liestal.

\section{DIE LANDSCHAFT ALS GANZES}

Der Exkursionsraum ist durch den landschaftlichen Gegensatz zwischen Hochrheintal und Birseck einerseits und Gempenplateau andererseits gekennzeichnet. Dort breite, hochwasserfreie, z. T. lößbedeckte Terrassen, fließende Gewässer, mildes, sonnenreiches Klima im Bereiche der Wärmeinsel des Rheingrabens, gute Durchgängigkeit; hier eine wellige Hochfläche mit vorwiegend armen Böden auf Hauptrogenstein und Malmkalk, mit Wassermangel, rauhem und regenreicherem Klima, abgetrennt von den umgebenden Tälern durch einen die Steilhänge überkleidenden Waldgürtel und nur schwer zugänglich.

In den begünstigten Talräumen hat die Besiedlung in vorgeschichtlicher Zeit eingesetzt, wie die paläo- und mesolithischen Stationen beweisen. Hier verlaufen die alten Straßen vom Oberrhein in die Zentral- und Westschweiz, hier war schon in keltorömischer Zeit eine relativ dicht besiedelte Kulturlandschaft entwickelt, während auf dem Gempenplateau in dieser Periode offenbar nur in der Gegend von Gempen und Nuglar kleine Rodungssiedlungen bestanden. In dieser Refugiallage vermochte sich die keltoromanische Bevölkerung noch längere Zeit über die Landnahmeperiode hinaus zu behaupten, während die Täler schon von den Alemannen besetzt waren. Die hochmittelalterliche Rodungsphase ließ auf dem Plateau und an seinen Abhängen neue Siedlungen entstehen (Hochwald, Seewen, St. Pantaleon). Seit Ende des Mittelalters erfuhr das Siedlungs- und Agrargefüge hier einen Ausbau durch die Gründung von Einzelhöfen in den von den Plateaudörfern entfernteren Bezirken; eine ältere Hofgeneration entstand vom 15. bis 18. Jahrhundert, eine jüngere seit der Aufhebung des Flurzwanges zu Beginn des letzten Jahrhunderts. Deren Blockfluren brachten ein neues Element in die traditionelle Agrarlandschaft mit ihren schmalstreifigen Gewannfluren.

Die Absonderung der Plateaulandschaft dokumentiert sich auch in der staatsräumlichen Entwicklung, wurde sie doch vom Kanton Solothurn erworben, der sogar in einer schmalen Zone zum rechten Birsufer bei Dornach vorstößt.

Die neuzeitliche Entwicklung der Kulturlandschaft hat die Differenzierung zwischen Plateau- und Tälerlandschaft noch verstärkt. Im günstig gelegenen und mit Wasserkraft versehenen Birseck und im Hochrheintal siedelte sich von Basel aus die Industrie an, sei es auf der durch die Birskorrektion geschützten untern Stufe der Niederterrasse oder aber in Bahnnähe und auf den Steinsalzvorkommen des Hochrheintales (Schweizerhalle). Unter dem Einfluß der Industriesiedlung und der Stadt Basel entwickelte sich die Bevölkerung progressiv, und es entstanden neben den altdörflichen Siedlungskernen ausgedehnte neue Wohnquartiere in lockerer Bauweise, deren Bewohner zum Teil in Basel arbeiten (Bevölkerungszunahme z.B. in Reinach von 1950-1960: $77 \%$ ). Damit war ein tiefgreifender Wandel der beruflichen und sozialen Struktur des Bevölkerungskörpers dieser Vorortgemeinden verbunden, deren überkommene Agrargesellschaft völlig durch eine suburbane Gesellschaftsstruktur mit Vorherrschen von Angestellten und Arbeitern ersetzt wurde. 
Anders verläuft die Entwicklung auf dem Gempenplateau. Die Einwohnerzahlen sind infolge der Landflucht vorwiegend rückläufig. Gempen und Hochwald besitzen noch eine überwiegend agrarische Struktur, und nichts deutet darauf hin, daß die stürmische wirtschaftliche Entwicklung der Talräume auf die Hochfläche übergreift. Innerhalb der Basler Region wird jedoch dem Plateau als zukünftigem Wohnraum für einen Teil der Bevölkerung der Basler Agglomeration Bedeutung zukommen.

1 Klima

\section{STATISTISCHER ANHANG}

\section{Temperaturen}

Basel-Binningen (Periode 19o1-1940) :

Jahresmittel $\quad 9,1^{\circ}$

Julimittel $\quad 18,1^{\circ}$

Januarmittel $\quad 0,5^{\circ}$

mittl. Jahresschwankung $\quad 17,6^{\circ}$

\section{Niederschläge}

(Jahresmittel, Periode 19o1-194o)

Neue Welt, $267 \mathrm{~m} \quad 836 \mathrm{~mm}$

Pfeffingen, $394 \mathrm{~m} \quad 927 \mathrm{~mm}$

Liestal, $325 \mathrm{~m} \quad 997 \mathrm{~mm}$

Grellingen, $328 \mathrm{~m} \quad 1023 \mathrm{~mm}$

Seewen, $551 \mathrm{~m} \quad 1014 \mathrm{~mm}$

\section{Gewässer}

Birs bei Münchenstein (Periode 1917-1960)

Mittlerer monatlicher Abfluß

Größtes Monatsmittel: März

Kleinstes Monatsmittel: August

Maximum des Abflusses: Mai $19+6$

Minimum des Abflusses: Juni 1921

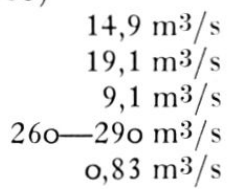

Arealstatistik 1952

3 Gemeindearcale (in ha)

Total

Kulturland

Höhe ü. M.

Arlesheim BL

Dornach SO

$\mathrm{m}$

Reinach BL

335

334

Grellingen $\mathrm{BE}$

325

\begin{tabular}{|c|c|c|c|c|c|}
\hline Muttenz BL & 292 & 1665 & 714 & 667 & 285 \\
\hline Pratteln BL & 298 & 1079 & 707 & 272 & 100 \\
\hline Gempen SO & 676 & 599 & 372 & 211 & 16 \\
\hline Hochwald SO & 620 & 842 & 465 & 353 & 24 \\
\hline Seewen SO & 552 & 1639 & 920 & 670 & 49 \\
\hline Büren $\mathrm{SO}$ & 447 & 623 & 334 & 260 & 29 \\
\hline Nuglar-St. Pantaleon SO & 492 & 634 & 374 & 235 & 25 \\
\hline
\end{tabular}

\section{Bodennutzung 1955}

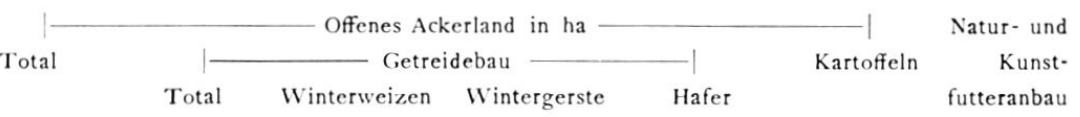

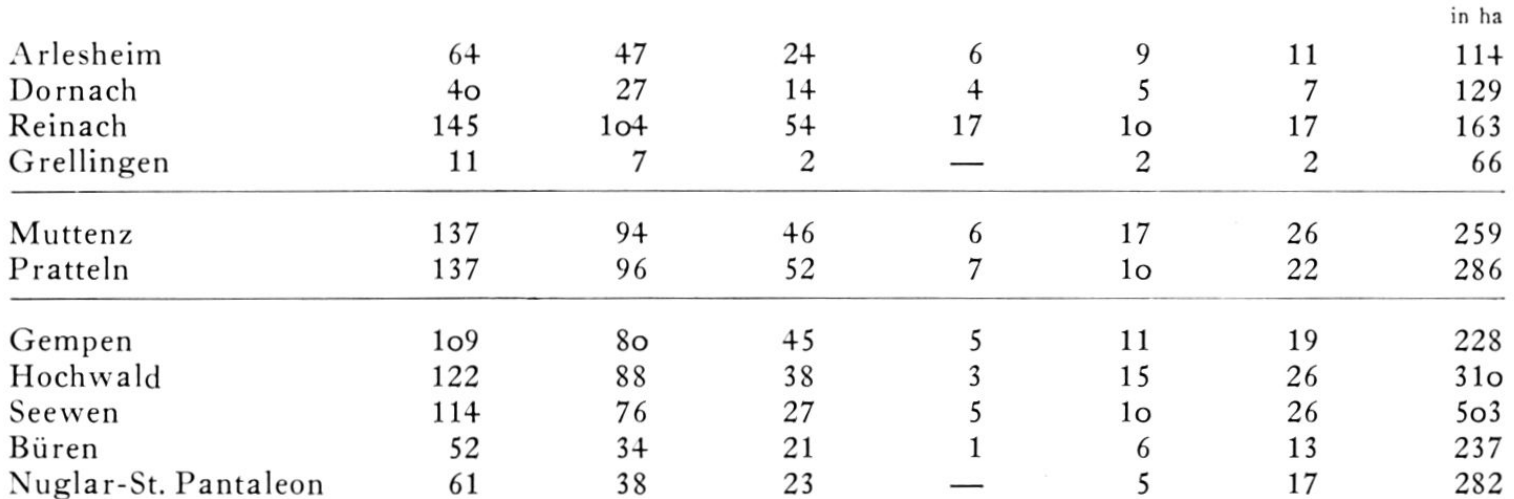




\begin{tabular}{|c|c|c|c|c|c|c|c|c|}
\hline 5 Bevölkerung & 1850 & 1880 & 1900 & 1930 & $19+1$ & 1950 & 1960 & $\begin{array}{c}\mathrm{Zu}-/ \text { Abnahme } \\
1850-1960 \\
\%\end{array}$ \\
\hline Arlesheim & 910 & 947 & 1599 & 3228 & 3360 & 3898 & 5219 & 474 \\
\hline Dornach & 840 & 1169 & 1543 & 3067 & 3056 & 3572 & 4260 & 4o7 \\
\hline Reinach & 816 & 951 & 1213 & 2558 & 2813 & 3475 & 6152 & 654 \\
\hline Grellingen & 512 & 1032 & 1012 & 1085 & 1067 & 1160 & 1399 & 173 \\
\hline Muttenz & $1322 *$ & 2057 & 2502 & 4966 & 5929 & 7125 & 11963 & 805 \\
\hline Pratteln & 1371 & 1868 & 2425 & +782 & $51+2$ & 6863 & 9492 & 592 \\
\hline Gempen & 378 & 376 & 355 & 345 & 308 & 301 & 250 & -34 \\
\hline Hochwald & 624 & 588 & 582 & 479 & 448 & 463 & 463 & -26 \\
\hline Seewen & 933 & 823 & 762 & 805 & 840 & 869 & 844 & -10 \\
\hline Büren & 611 & 559 & 546 & 531 & 568 & 548 & 561 & -8 \\
\hline Nuglar-St. Pantaleon & 658 & 657 & 666 & 775 & 794 & 815 & 855 & 30 \\
\hline
\end{tabular}

* Muttenz 18502222 E., abzüglich 9oo E. von Birsfelden, das $187+$ von Muttenz getrennt wurde.

6 Berufstätige 1950

\begin{tabular}{|c|c|c|c|c|c|c|c|c|c|}
\hline ufstätige 1950 & $\mathrm{~L}^{*}$ & $\%$ & $\mathrm{I}+\mathrm{H}^{*}$ & $0 \%$ & $\mathrm{G}, \mathrm{V}^{*}$ & $\%$ & $\mathrm{Ue}^{*}$ & $\%$ & $\begin{array}{l}\text { Total } \\
=100^{\circ} \%\end{array}$ \\
\hline Arlesheim & 56 & 3 & 845 & 46 & 415 & 23 & $50 t$ & 28 & 1820 \\
\hline Dornach & 48 & 3 & 965 & 55 & 313 & 18 & 416 & 24 & $17+2$ \\
\hline Reinach & 79 & 5 & 885 & 56 & 384 & 24 & 227 & 15 & 1575 \\
\hline Grellingen & 24 & 4 & 383 & 69 & 98 & 18 & 49 & 9 & 554 \\
\hline Muttenz & 98 & 3 & 1609 & 50 & 1037 & 32 & 467 & 15 & 3211 \\
\hline Pratteln & 105 & 3 & 1961 & 63 & 685 & 22 & 376 & 12 & 3127 \\
\hline Gempen & 66 & 42 & 65 & 42 & 15 & 10 & 1o & 6 & 156 \\
\hline Hochwald & 97 & 48 & 76 & 38 & 15 & 7 & 15 & 7 & 203 \\
\hline Seewen & 135 & 35 & 188 & 50 & 24 & 6 & 34 & 9 & 381 \\
\hline Büren & 96 & 32 & 167 & 56 & 23 & 8 & 11 & 4 & 297 \\
\hline Nuglar-St. Pantaleon & 71 & 18 & 245 & 64 & 47 & 12 & 23 & 6 & 386 \\
\hline
\end{tabular}

* $\mathrm{L}=$ Landwirtschaft $\mathrm{I}+\mathrm{H}=$ Industrie und Handwerk $\mathrm{G}, \mathrm{V}=$ Gastgewerbe, Handel und Verkehr $\mathrm{Ue}=$ andere Berufe.

7 Pendler ausgewählter Gemeinden 1950

\begin{tabular}{|c|c|c|c|c|c|c|c|}
\hline \multirow{4}{*}{$\begin{array}{l}\text { Arlesheim } \\
\text { Dornach }\end{array}$} & \multirow{4}{*}{$\begin{array}{l}\quad 1 \\
\text { Berufstätige } \\
1820 \\
1742\end{array}$} & \multirow{2}{*}{\multicolumn{2}{|c|}{$\begin{array}{c}2 \\
\text { Einpendler } \\
0 \% \text { von } 1\end{array}$}} & \multirow{2}{*}{\multicolumn{2}{|c|}{$\begin{array}{r}4 \quad 5 \\
\text { Auspendler } \\
\% \text { von } 1\end{array}$}} & \\
\hline & & & & & & $\begin{array}{c}\text { Ausp } \\
\text { Basel }\end{array}$ & $\begin{array}{l}\text { endler nach } \\
\% \text { von } 4\end{array}$ \\
\hline & & 559 & 30,7 & 735 & 40,4 & 484 & 65,8 \\
\hline & & 668 & 38,3 & 559 & 32,0 & 267 & 47,8 \\
\hline Muttenz & 3211 & 1279 & 39,8 & 1471 & 45,8 & 1082 & 73,5 \\
\hline Pratteln & 3127 & 2031 & 64,9 & 769 & 24,6 & 376 & 49,0 \\
\hline Gempen & 156 & o & 0,0 & 63 & 40,4 & 15 & 23,8 \\
\hline Hochwald & 203 & o & 0,0 & 55 & 27,1 & 13 & 23,6 \\
\hline
\end{tabular}

\section{ÜBER DILUVIALE ABLAGERUNGEN AUS DER UMGEBUNG VON CHIASSO}

\section{HANS RENFER}

Der vorliegende Bericht stellt die Zusammenfassung des glaziologischen Teiles einer im Entstehen begriffenen Arbeit über die V.di Muggio dar. Auf Grund zahlreicher neuer und z.T. tiefreichender Aufschlüsse konnte nachgewiesen werden, daß die Diluvialablagerungen im Mendrisiotto älter sind, als bisher allgemein angenommen wurde, und daß die jüngste Eiszeit nur noch durch vereinzelte Moränen mit Sicherheit nachgewiesen werden kann.

Der als mindeleiszeitlich zu datierende Ferretto stellt im Varesotto und Mendrisiotto die älteste nachweisbare Quartärablagerung dar. Nach NANgERoni (1954) ist E Bizzòzero (3 km SE Varese) am Abhang gegen die Olona auf oligo-/miozäner Molasse 